\title{
One-Pot Synthesis of Trifluoromethylated Quinazolin-4(3H)-ones with Trifluoroacetic Acid as $\mathrm{CF}_{3}$ Source
}

\author{
Sofia Almeida, ${ }^{\dagger, \S, \ddagger}$ Roger Marti, ${ }^{\ddagger}$ Ennio Vanoli, ${ }^{\ddagger}$ Stefan Abele, ${ }^{\dagger}$ and Simone Tortoioli* ${ }^{\dagger} \dagger$ \\ ${ }^{\dagger}$ Chemical Development, Idorsia Pharmaceuticals Ltd., Hegenheimermattweg 91, 4123 Allschwil, Switzerland \\ ${ }^{\ddagger}$ HES-SO, University of Applied Sciences Western Switzerland, Haute Ecole d'Ingénierie et d'Architecture Fribourg, Institute of \\ Chemical Technology, Boulevard de Pérolles 80, CH-1700 Fribourg, Switzerland
}

Supporting Information

ABSTRACT: A novel and convenient one-pot sequential cascade method for the preparation of 2-trifluoromethylquinazolin-4(3H)-ones is described. Trifluoroacetic acid (TFA) was employed as inexpensive and readily available $\mathrm{CF}_{3}$ source, which in the presence of T3P was condensed with a variety of anthranilic acids and amines to provide the products in up to $75 \%$ yield. The protocol was proved to be robust on $80 \mathrm{~g}$ scale, and the synthetic versatility of the prepared quinazolinon-4ones was demonstrated by derivatization to further useful building blocks.

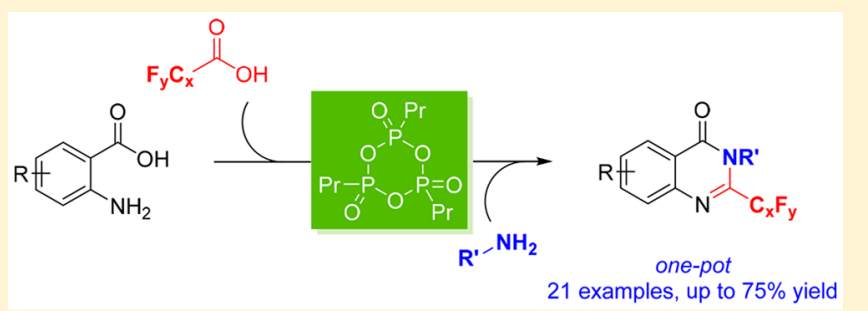

ypyridines, ${ }^{7 \mathrm{a}, \mathrm{b}}$ pyrimidine derivatives, ${ }^{7 \mathrm{c}} \beta$-carbolines ${ }^{7 \mathrm{~d}}$ and 2 (trifluoromethyl)quinazolin-4- $(3 H)$-one $(11) .^{7 \mathrm{e}}$

Quinazolin-4(3H)-ones are a class of compounds present in naturally occurring alkaloids as well as in commercial drugs and displaying a broad spectrum of biological activities. ${ }^{8}$

Surprisingly, among the vast literature around quinazolinones and their synthetic accesses, ${ }^{8 \mathrm{e}}$ a survey on the available methods for the corresponding 2-trifluoromethyl substituted scaffolds 4 revealed a much more limited number of possibilities (Scheme 1). Anthranilic amides (I) are among the substrates preferentially employed using ethyl trifluoroacetate in sodium ethoxide, ${ }^{9 a}$ trifluoroacetic anhydride, ${ }^{6 c}$ or in one specific example, with TFA under microwave heating as $\mathrm{CF}_{3}$-reagent. ${ }^{7 \mathrm{e}}$ Anthranilic esters (II) have also been condensed with unstable trifluoroacetamidine, ${ }^{9 b, c}$ while nitrile derivatives (III) have been reported to cyclize with trifluoroacetic anhydride. ${ }^{9 \mathrm{~d}}$ One specific example is reported in which 2-(trifluoromethyl)quinazolines were obtained in few steps from alkyne (IV) via hydroamination, ${ }^{9}$ while the last approach relied on first reacting isatoic anhydride $(\mathbf{V})$ with trifluoroacetic anhydride to generate 2-trifluoromethylbenzoxazin-4-ones 2 , followed by displacement with amines. ${ }^{9 f}$

At the beginning of our study, we aimed to have a more general and straightforward access to 2-trifluoromethylquinazolinones 4 that offers opportunities for diversification by using the commodity chemical TFA as trifluoromethyl source. Anthranilic acids $\mathbf{1}$ being simpler and more available raw material as compared to amides, esters, anhydrides and cyanides, are the substrates of choice. For this purpose, we envisioned to use T3P as both coupling and condensation

Received: February 16, 2018

Published: April 12, 2018 
Scheme 1. Strategies for the Synthesis of 2Trifluoromethylquinazolin-4(3H)-ones Derivatives

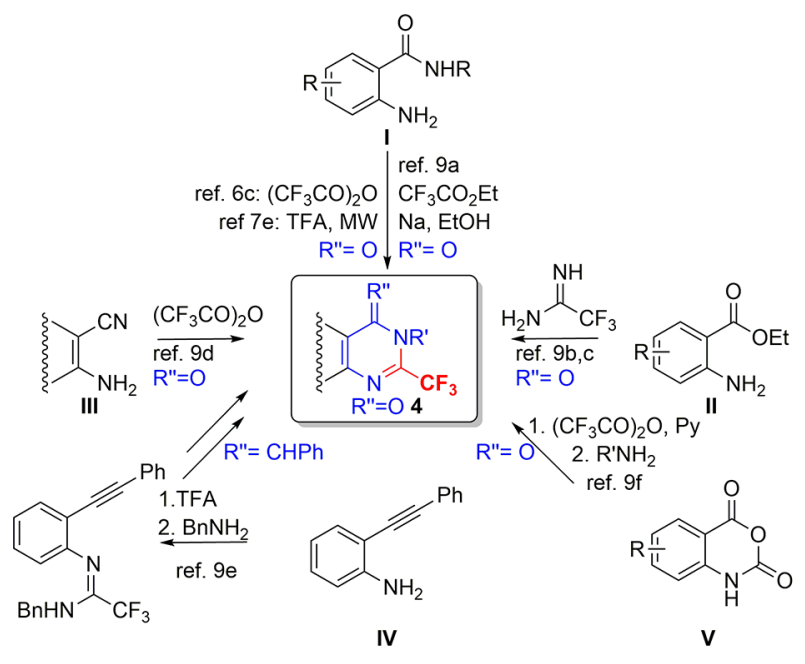

more classical protocol that uses trifluoroacetic anhydride as both $\mathrm{CF}_{3}$ source and condensation partner. ${ }^{12,9 f}$

To prepare $\mathrm{CF}_{3}$-quinazolin-4- $(3 \mathrm{H})$-ones 4 with the same precursors and similar one-pot strategy, we could first transform the acid moiety into an amide 3 , followed by $\mathrm{N}$ trifluoroacetylation and dehydration (strategy I, Scheme 3 ) or alternatively, $\mathrm{N}$-trifloroacetylation could be followed by amide formation and cyclization (strategy II, Scheme 3). With trifluoroacetic anhydride leading to the corresponding benzoxazinones 2 (Scheme 2), ${ }^{9 f, 12}$ we envisaged to use T3P as alternative and afford the synthesis of $\mathrm{CF}_{3}$-quinazolinones either with strategy I or II.

Using 2-aminobenzoic acid (1a) as substrate for the initial screening of reaction conditions and simple ammonia or $\mathrm{NH}_{4} \mathrm{Cl}$ as the amine counterpart, no primary amide was detected with strategy I even after prolonged stirring and heating in toluene. ${ }^{13}$ Pleasingly, by replacing ammonia with benzylamine, conversion into the corresponding benzylamide 3a occurred, which after treatment with a mixture of T3P and TFA was transformed into the $\mathrm{N}$-trifluoroacetyl derivative and subsequently cyclized to the desired quinazolinone in $70 \%$ isolated yield (strategy I Scheme 3, see Experimental Section for details).

Under these conditions, a wide range of substrates were prepared (Scheme 4).

Electron-rich anthranilic acids afforded quinazolinones $\mathbf{4 b}$ and $4 \mathrm{c}$ in good yields, as well as halogen-containing products $\mathbf{4 d - h}$ that were prepared in $51-72 \%$ yields. The highly electron-withdrawing trifluoromethyl-group was also well tolerated and the corresponding product $4 \mathbf{i}$ was obtained in $72 \%$ yield.

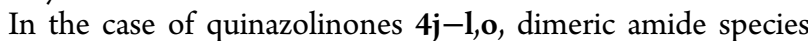
deriving from the attack of the nucleophilic amino moiety of an anthranilic acid $\mathbf{1 j}-\mathbf{l}, \mathbf{o}$ to the T3P-activated acid moiety of another molecule of $\mathbf{1 j}-\mathbf{l}, \mathbf{o}$ were prevalently formed with strategy I during the amidation step (Scheme 5). ${ }^{14}$

The observed higher reactivity of the aniline function for those substrates suggested that reverting the order of events (strategy II Scheme 3) would suppress the formation of this side-product. In such way products $4 \mathbf{j}-\mathbf{l}, \mathbf{o}$ were obtained in $38-75 \%$ yields (see Experimental Section for details). Our synthetic approach bears as well some limitations and pyridinecontaining bicycles $\mathbf{4 m}, \mathbf{n}$ were obtained only in modest to low yields with strategy I. In addition, 5-aminoorotic acid and 2aminobenzensulfonic acid did not afford desired products $4 p$ and $\mathbf{4 q}$, most likely due to a lack of reactivity of the sulfonic acid and incompatibility of T3P with 5-aminoorotic acid, respectively.

The possibility to modify both the amine and acid counterparts offers the advantage of chemical diversification which is especially valuable in combinatorial and exploratory chemistry. Representative modified substrates were prepared according to strategy I (Scheme 6). Quinazolinone 4r deriving from challenging hindered $o$-toluidine was obtained in $42 \%$ yield, while with $n$-pentylamine the corresponding product $4 \mathrm{~s}$ was prepared in $59 \%$ yield. By using L-leucine methyl ester as amine, instead of the desired product, benzo[1,4] diazepine-2,5dione 6 was obtained in a not-optimized $32 \%$ yield. ${ }^{15}$ This result demonstrates that anthranilic acids in combination with T3P are a practical synthetic equivalent of isatoic anhydride $\mathbf{V}$, known to condense with $\alpha$-amino acids for the preparation of benzo[1,4] diazepine-2,5-diones. ${ }^{15 a-f}$ With regards to the acid partner, variation was achieved by using commercially available 
Scheme 3. T3P-Mediated Synthesis of 3-Benzyl-2-(trifluoromethyl)quinazolin-4(3H)-ones 4

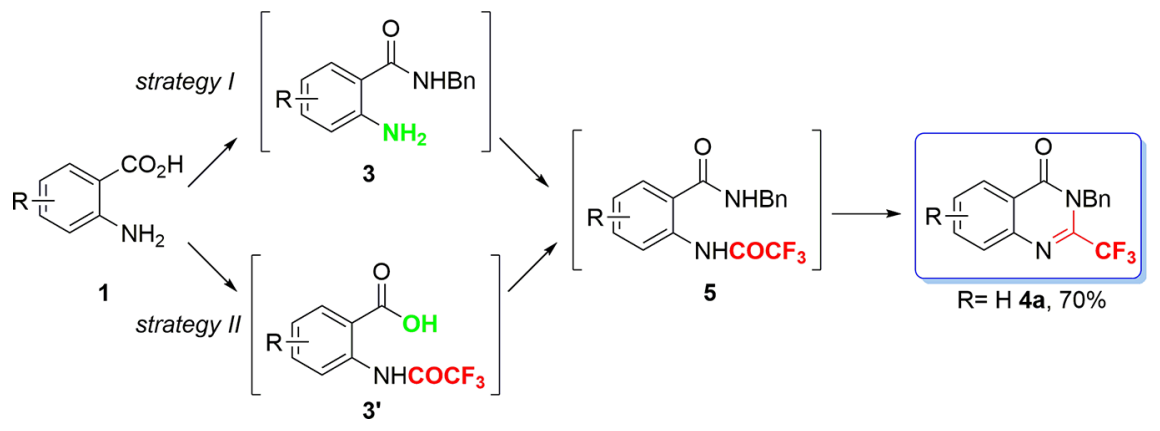

Scheme 4. $\mathrm{CF}_{3}$-Quinazolinones 4 Prepared by Variation of Anthranilic Acid Substrates<smiles>COc1cccc2nc(C(F)(F)F)[nH]c(=O)c12</smiles><smiles>COc1ccc2c(=O)n(Cc3ccccc3)c(C(F)(F)F)nc2c1</smiles><smiles></smiles>

bb $69 \%$<smiles>O=c1c2cc(I)ccc2nc(C(F)(F)F)n1Cc1ccccc1</smiles><smiles></smiles>

$4 g, 67 \%$<smiles>O=c1c2ccc([N+](=O)[O-])cc2nc(C(F)(F)F)n1Cc1ccccc1</smiles><smiles>O=c1c2cc3ccccc3cc2nc(C(F)(F)F)n1Cc1ccccc1</smiles>

4d, $72 \%$<smiles>O=c1[nH]c(C(F)(F)F)nc2c(Cl)cccc12</smiles><smiles>O=c1c2cc(F)ccc2nc(C(F)(F)F)n1Cc1ccccc1</smiles><smiles></smiles>

$4 h, 59 \%$

$4 i, 72 \%$

\section{$4 j, 75 \%$}

$4 k, 55 \%$<smiles></smiles><smiles>O=c1c2cccnc2nc(C(F)(F)F)n1Cc1ccccc1</smiles>

$4 I, 48 \%$

$4 \mathrm{~m}, 25 \%$<smiles>O=c1c2cc(Br)cnc2nc(C(F)(F)F)n1Cc1ccccc1</smiles>

$4 n, 21 \%$<smiles>O=c1c2ccncc2nc(C(F)(F)F)n1Cc1ccccc1</smiles>

$40,38 \%$<smiles>O=c1[nH]c(=O)c2[nH]c(=O)n(Cc3ccccc3)c(C(F)(F)F)nc1=2</smiles>

$4 p, 0 \%$<smiles>O=S1(=O)c2ccccc2N=C(C(F)(F)F)C1Cc1ccccc1</smiles>

$4 q, 0 \%$
Scheme 5. Dimeric Species Formation

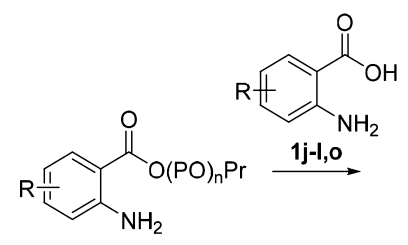

1j-1,0 T3P-adducts

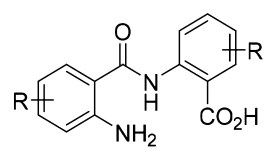

dimers chlorodifluoroacetic acid and pentafluoropropionic acid as examples, allowing for the preparation of the corresponding quinazolinones $\mathbf{4 t}, \mathbf{u}$ in reasonable yields.

Our developed protocol is also amenable to the use of amides (Scheme 7). Pyrazole carboxamide 7 was smoothly converted into quinazolinone $\mathbf{8}$, a trifluoromethylated variant of the core structure of sildenafil. Additionally, 2-aminobenzenesulfonamide 9 was converted into 3-(trifluoromethyl)benzothiadiazine-1,1-dioxide 10, albeit in low isolated yield due to instability of the product during the aqueous workup hydrolyzing back to the starting material.

To prove the practicality and robustness of our T3Pmediated method, we performed a scale-up of the reaction to $80 \mathrm{~g}$ of anthranilic acid 1a affording $86.5 \mathrm{~g}$ of pure quinazolinone $4 \mathrm{a}$ corresponding to $50 \%$ yield. The lower
Scheme 6. Amine and Fluoride Source Variation<smiles>Cc1ccccc1-n1c(C(F)(F)F)nc2ccccc2c1=O</smiles>

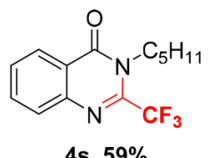

$4 r, 42 \%$ $4 \mathrm{~s}, 59 \%$<smiles>CC(C)CC1NC(=O)c2ccccc2NC1=O</smiles><smiles>C=CC=[Nb]</smiles>

$1 \mathbf{a}$

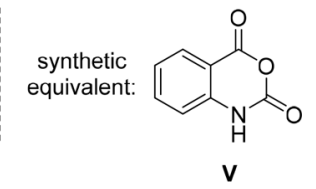<smiles></smiles><smiles>O=c1c(Br)nc2ccccc2nc1C(F)(F)F</smiles>

$4 \mathrm{t}, 49 \%$

$4 \mathrm{u}, 46 \%$

yield compared to the small scale result (70\%, Scheme 3$)$ is caused by the different isolation procedure (see Experimental Section for details). On $80 \mathrm{~g}$ scale, classical flash chromatography was replaced by trituration with TBME and a significant amount of product was lost into the mother liquors after 
Scheme 7. Synthesis of 5-Trifluoromethylpyrimidin-7-ones 8 and 3-(Trifluoromethyl)benzothiadiazine-1,1-dioxide 10 from Corresponding Amides
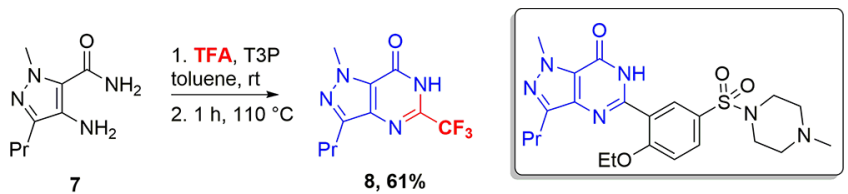

sildenafil (Viagra)

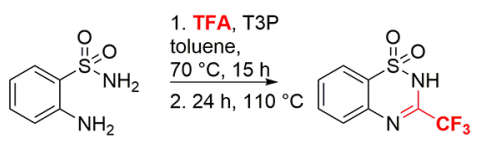

$10,23 \%$

filtration, indicating room for improvement in the final recovery by fine-tuning of the trituration conditions.

Finally, the synthetic versatility of the obtained $\mathrm{CF}_{3}$ quinazolinones prepared was demonstrated (Scheme 8). Debenzylation with $\mathrm{AlCl}_{3}$ followed by chlorination-aromatization with $\mathrm{POCl}_{3}$ transformed $4 \mathrm{a}$ into 4-chloro-2-trifluoromethylquinazine 12 in high yield. This versatile intermediate was successfully converted into different derivatives through functionalization at the highly reactive 4-position. For instance, $\mathrm{C}-\mathrm{C}$ bond formation was achieved by treatment with $i \mathrm{PrMgCl}$ resulting in the formation of alkylated product 13 in high yield. $S_{N} A r$ with aniline 14 smoothly produced derivative 15 in $71 \%$ yield and methoxylation with $\mathrm{NaOMe}$ afforded derivative 16 in quantitative yields.

The highly reactive nature of TFA combined with T3P prompted us to investigate the thermal stability of the mixture-information that becomes of vital importance for preparation and handling on scale. Differential scanning calorimetry (DSC) is a diagnostic and very effective measurement that is usually performed for this purpose, and the result of the nonisothermal DSC of the mixture TFA-T3P in toluene is shown in Figure 1.

Fortunately, only minor exothermic events appeared at lower temperatures (left limits of 118 and $196^{\circ} \mathrm{C}$ ), and due to the low amount of energy involved they can be regarded as no safety concern. Above $254{ }^{\circ} \mathrm{C}$, larger decompositions are detected with release of moderate to high energies, confirming that this mixture is stable and can be handled and safely used at temperatures below $100{ }^{\circ} \mathrm{C}$. This finding is well in line with the known high thermal stability of T3P itself. $^{16}$

\section{CONCLUSIONS}

In summary, we have developed a novel and practical one-pot sequential cascade method for the preparation of 2-trifluoromethylquinazolin-4(3H)-ones 4. Our approach uses inexpensive TFA as $\mathrm{CF}_{3}$ source in combination with commercially available T3P as coupling and dehydrating agent. Chemical diversification was achieved by varying the amine and the acid partners and represents an additional strength of the current method. Most of the compounds that have been prepared are novel (with the exception of $2 a, b, 4 a, 6,11$ and 12). The potential interest for this specific class of quinazolinones does not only rely on the similarity to the corresponding biologically active nonfluorinated analogs, but also on the fact that such building blocks can be easily derivatized providing access to a wide range of trifluoromethylated derivatives that might be difficult to access with other methods.

\section{EXPERIMENTAL SECTION}

General Methods. All the chemicals employed are commercially available and were used as such with no further purification. Solution of T3P in toluene was purchased from Euticals.

Infrared spectra were recorded on a PerkinElmer SPECTRUM ONE-Spectrophotometer and are reported as $\mathrm{cm}^{-1}$ ( $\mathrm{w}=$ weak, $\mathrm{m}=$ medium, $s=$ strong). High resolution mass spectrometric measurements were performed with SYNAPT G2MS (Waters) Q-Tof instrument that can provide up to $40000 \mathrm{fwhm}$ resolution, dataacquisition rate of 20 spectra/second, exact mass ( 1 ppm RMS) information and a dynamic range of up to 5 orders of magnitude (conditions for analysis in the Supporting Information). LC-MS analyses were performed using Aquity Waters system equipped with an Agilent G4220A binary pump coupled with Thermo Finnigan MSQ Plus MS (Ionization: ESI+), Agilent DAD-G4212A and Column oven Dionex TCC-3200. (conditions for analysis in the Supporting Information). Melting points were measured with DSC analyses. ${ }^{1} \mathrm{H}$ and ${ }^{13} \mathrm{C}$ (proton decoupled) spectra were recorded on a Bruker NMR $500 \mathrm{MHz}$ spectrometer Avance HD equipped with DCH-Cryoprobe (500 and $125 \mathrm{MHz}$ respectively) and with a Bruker NMR $400 \mathrm{MHz}$

Scheme 8. Derivatization of 3-Benzyl-2-(trifluoromethyl)quinazolin-4(3H)-one (4a)

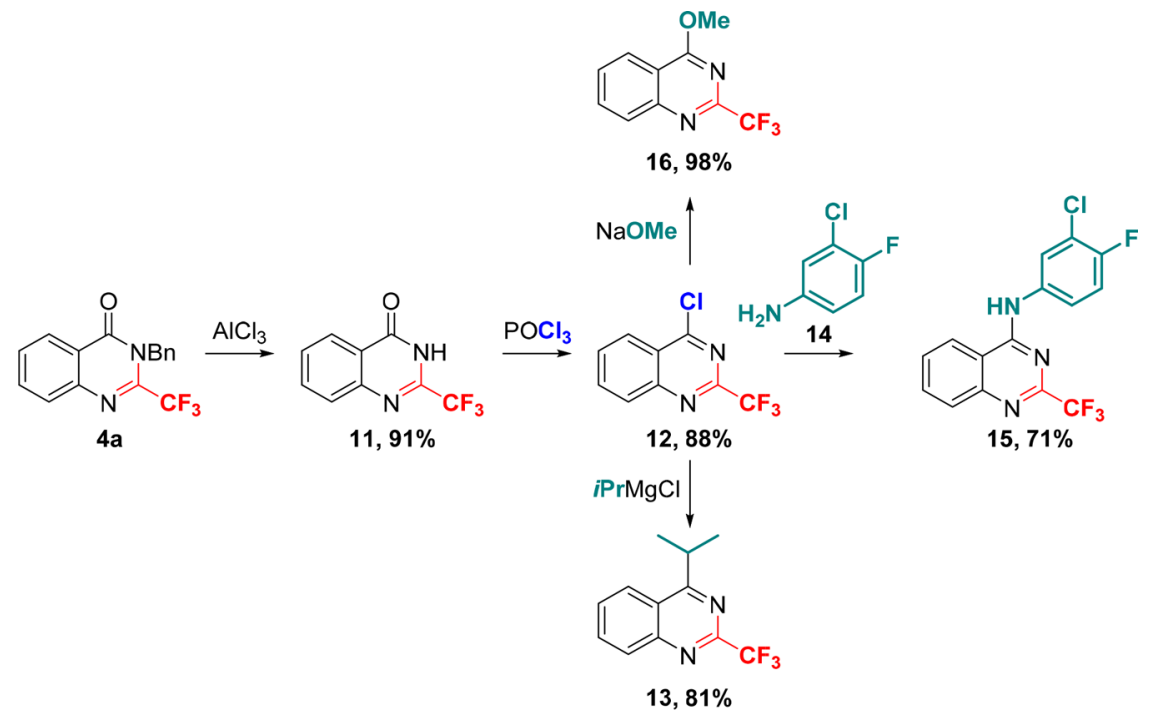




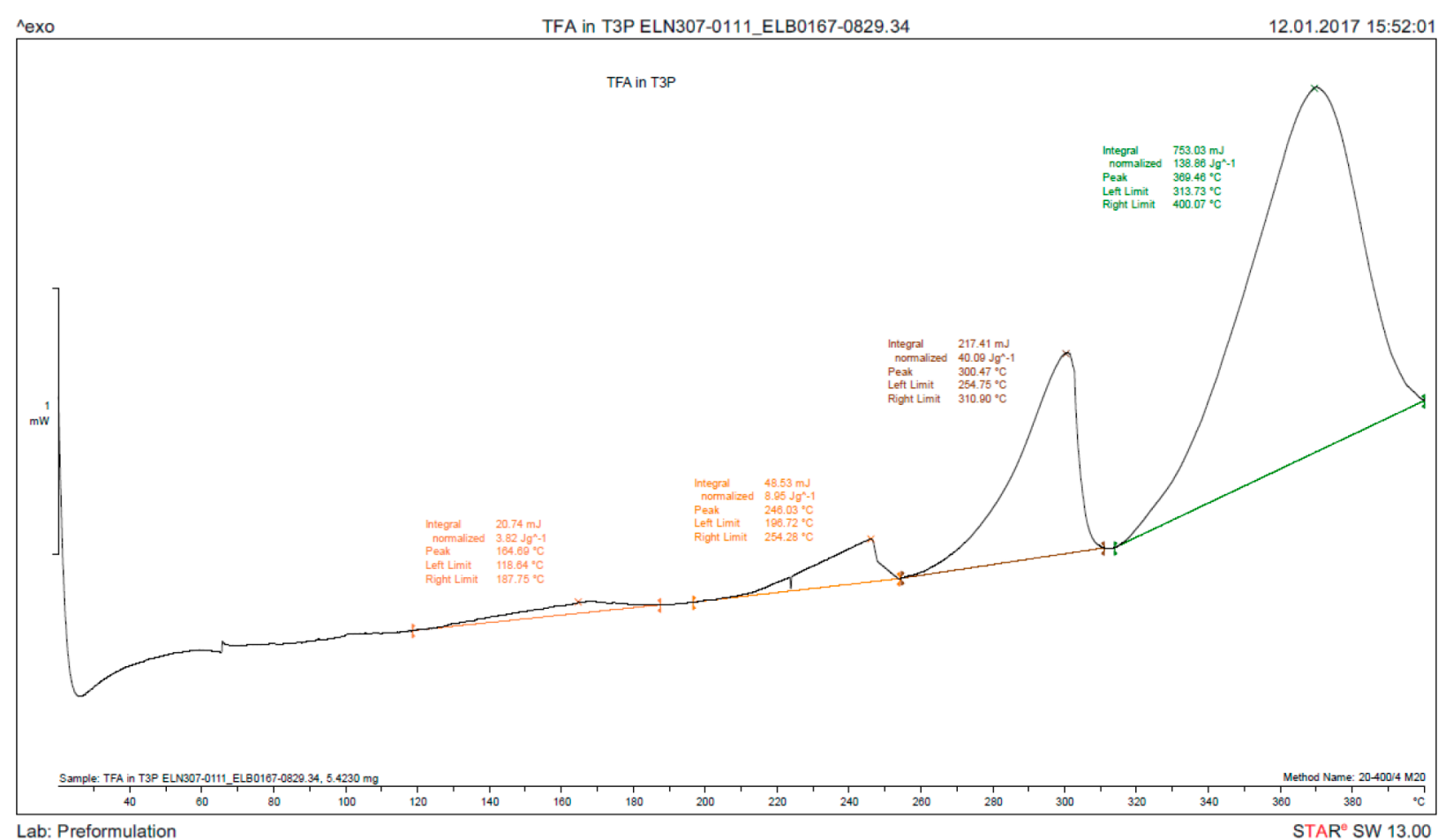

Figure 1. DSC of TFA-T3P mixture in toluene (heat ramp $4{ }^{\circ} \mathrm{C} / \mathrm{min}$ ).

Spectrometer Avance 2 used as well to measure ${ }^{19} \mathrm{~F}$ spectra recorded with $5 \mathrm{~mm}$ BBO Probehead at $375 \mathrm{MHz}$. Chemical shifts $(\delta)$ values are reported in parts per million using residual solvent signal as reference and the coupling constants $(J)$ are reported in Hz. Novel compounds were characterized with ${ }^{1} \mathrm{H},{ }^{13} \mathrm{C}$ NMR, IR and HRMS and melting point when applicable.

General Procedure for 2-Trifluoromethylbenzoxazin-4-ones 2. To a suspension of the corresponding anthranilic acids $\mathbf{1 a}, \mathbf{b}(1.0 \mathrm{~g}$, 1.0 equiv) in toluene $(4 \mathrm{~mL})$ at $\mathrm{rt}$ was added dropwise trifluoroacetic acid ( 1.15 equiv) followed by T3P $(50 \% \mathrm{w} / \mathrm{w}$ in toluene, 1.2 equiv). The reaction mixture was stirred at $\mathrm{rt}$ for $2 \mathrm{~h}$ before adding $\mathrm{Et}_{3} \mathrm{~N}(1.0$ equiv) and T3P ( $50 \%$ in toluene, 1.0 equiv). The reaction mixture was then heated at reflux $\left(110-115{ }^{\circ} \mathrm{C}\right)$. After completion of the reaction, the mixture was cooled to $\mathrm{rt}$ and quenched by slow addition of aq. sat. $\mathrm{NaHCO}_{3}(25 \mathrm{~mL}$, Caution! Gas evolution) followed by extraction with solvents such as EtOAc or DCM. Collected organic phases were washed with aq. sat. $\mathrm{NaHCO}_{3}(25 \mathrm{~mL})$ and brine $(25 \mathrm{~mL})$, dried over anhydrous $\mathrm{MgSO}_{4}$, filtered and evaporated under reduced pressure to afford desired crude benzoxazinones 2 .

2-(Trifluoromethyl)-4H-benzo[d][1,3]oxazin-4-one $(2 a) .{ }^{11 b}$ The reaction was performed according to the general procedure with anthranilic acid 1a as starting material. After workup, desired compound 2a was obtained without further purification as an offwhite solid (2.59 g, 83\%). mp $49{ }^{\circ} \mathrm{C} .{ }^{1} \mathrm{H}$ NMR $\left(400 \mathrm{MHz}, \mathrm{CDCl}_{3}\right) \delta$ $8.32\left(\mathrm{dd}, J_{1}=7.9 \mathrm{~Hz}, J_{2}=1.3 \mathrm{~Hz}, 1 \mathrm{H}\right), 7.94-7.99(\mathrm{~m}, 1 \mathrm{H}), 7.82(\mathrm{~d}, J$ $=7.6 \mathrm{~Hz}, 1 \mathrm{H}), 7.73\left(\mathrm{td}, J_{1}=7.8 \mathrm{~Hz}, J_{2}=1.1 \mathrm{~Hz}, 1 \mathrm{H}\right) .{ }^{13} \mathrm{C} \mathrm{NMR}(125$ $\left.\mathrm{MHz}, \mathrm{CDCl}_{3}\right) \delta 156.5,147.4(\mathrm{q}, J=42.0 \mathrm{~Hz}), 144.0,137.4,130.9$, $129.3,128.3,117.9,116.1(\mathrm{q}, J=276.0 \mathrm{~Hz}) .{ }^{19} \mathrm{~F}$ NMR $(375 \mathrm{MHz}$, $\left.\mathrm{CDCl}_{3}\right) \delta-72.49$. MS $[\mathrm{M}-\mathrm{H}]^{+}+\mathrm{H}_{2} \mathrm{O}$ 232.09. The analytical data are consistent with reported data.

7-Chloro-2-(trifluoromethyl)-4H-benzo[d][1,3]-oxazin-4-one (2b). ${ }^{11}$ The reaction was performed according to the general procedure with 4-chlorobenzoic acid $\mathbf{1 b}$ as starting material. The crude compound was purified by trituration with TBME yielding compound $2 \mathbf{b}$ as a light brown solid $(1.27 \mathrm{~g}, 89 \%)$. mp $50{ }^{\circ} \mathrm{C} .{ }^{1} \mathrm{H}$ $\operatorname{NMR}\left(500 \mathrm{MHz}, \mathrm{CDCl}_{3}\right) \delta 8.24(\mathrm{~d}, J=8.5 \mathrm{~Hz}, 1 \mathrm{H}), 7.81(\mathrm{~d}, J=2.0$ $\mathrm{Hz}, 1 \mathrm{H}), 7.69\left(\mathrm{dd}, J_{1}=2.0 \mathrm{~Hz}, J_{2}=8.5 \mathrm{~Hz}, 1 \mathrm{H}\right) .{ }^{13} \mathrm{C}$ NMR $(125$ $\left.\mathrm{MHz}, \mathrm{CDCl}_{3}\right) \delta 155.72,148.6(\mathrm{q}, J=42 \mathrm{~Hz}), 145.03,144.09,131.56$, $130.50,128.23,117.05,115.87(\mathrm{q}, J=166.6 \mathrm{~Hz}) .{ }^{19} \mathrm{~F}$ NMR $(375 \mathrm{MHz}$,
$\left.\mathrm{CDCl}_{3}\right) \delta-72.56$. MS $[\mathrm{M}-\mathrm{H}]^{+}+\mathrm{H}_{2} \mathrm{O}$ 266.00. The analytical data are consistent with reported data.

General Procedure for 3-Benzyl-2-(trifluoromethyl)quinazolin-4(3H)-ones $4 a-i, m, n, r-u$ (Strategy I). To a suspension of the corresponding anthranilic acids $\mathbf{1 a}-\mathbf{i}, \mathbf{m}, \mathbf{n}, \mathbf{r}-\mathbf{u}(1 \mathrm{~g}, 1.0$ equiv) in toluene $(4 \mathrm{~mL})$ at $\mathrm{rt}$ was added $\mathrm{Et}_{3} \mathrm{~N}$ (1.0 equiv) and $\mathrm{T} 3 \mathrm{P}$ $(50 \% \mathrm{w} / \mathrm{w}$ in toluene, 1.0 equiv). After stirring until total consumption of $\mathbf{1 a}-\mathbf{i}, \mathbf{m}, \mathbf{n}, \mathbf{r}-\mathbf{u}$ (checked by LC-MS, 3-5 h typically) benzylamine (0.98 equiv) was added dropwise at $\mathrm{rt}$ and then heated at $70{ }^{\circ} \mathrm{C}$ and left at this temperature for $12-15 \mathrm{~h}$. Aside, a solution of the fluorinated acid (1.0 equiv) in T3P (50\% w/w in toluene, 1.0 equiv) was prepared and added dropwise to the reaction mixture cooled to rt. The resulting mixture was stirred until total consumption of the amide (2-3 h typically), and then heated at reflux for $16-20 \mathrm{~h}$ until full conversion was achieved. The cooled reaction mixture was quenched by dropwise addition of aq. sat. $\mathrm{NaHCO}_{3}(25 \mathrm{~mL}$, Caution! Gas evolution) followed by extraction with solvents such as EtOAc/iPrOAc or DCM $(1 \times 25 \mathrm{~mL})$. Collected organic phases were washed with 25 $\mathrm{mL}$ of aq. sat. $\mathrm{NaHCO}_{3}$ and brine $(25 \mathrm{~mL})$ respectively, dried over anhydrous $\mathrm{MgSO}_{4}$, filtered and evaporated under reduced pressure to afford desired crude quinazolino-4-ones $4 \mathbf{a}-\mathbf{i}, \mathbf{m}, \mathbf{n}, \mathbf{r}-\mathbf{u}$.

3-Benzyl-2-(trifluoromethyl)quinazolin-4(3H)-one $(4 a))^{9 e}$ The reaction was performed according to the general procedure with anthranilic acid 1a as starting material, benzylamine and trifluoroacetic acid. The crude compound was purified by flash chromatography ( $n$ heptane/EtOAc: $100 / 0$ to $70 / 30$ ) yielding compound $\mathbf{4 a}$ as a white solid (1.54 g, 70\%). mp $95{ }^{\circ} \mathrm{C} .{ }^{1} \mathrm{H}$ NMR (400 MHz, $\left.\mathrm{CDCl}_{3}\right) \delta 8.37$ $(\mathrm{d}, J=8.0 \mathrm{~Hz}, 1 \mathrm{H}), 7.89(\mathrm{~d}, J=3.8 \mathrm{~Hz}, 2 \mathrm{H}), 7.63-7.70(\mathrm{~m}, 1 \mathrm{H})$, $7.33(\mathrm{~m}, 3 \mathrm{H}), 7.20(\mathrm{~d}, J=7.4 \mathrm{~Hz}, 2 \mathrm{H}), 5.47(\mathrm{~s}, 2 \mathrm{H}) .{ }^{13} \mathrm{C}$ NMR $(125$ $\left.\mathrm{MHz}, \mathrm{CDCl}_{3}\right) \delta 161.6,145.1,142.4(\mathrm{q}, J=35.9 \mathrm{~Hz}), 135.5,135.2$, $129.5,128.7,128.6,127.7,127.4,126.3,121.9,118.18$ (q, $J=277.2$ $\mathrm{Hz}), 47.90 .{ }^{19} \mathrm{~F} \mathrm{NMR}\left(375 \mathrm{MHz}, \mathrm{CDCl}_{3}\right) \delta-65.30$. MS $[\mathrm{M}+\mathrm{H}]^{+}$ $305.20 \mathrm{~m} / z$. The analytical data are consistent with reported data.

Scale-up Experiment. The reaction was performed in a $4 \mathrm{~L}$ double-jacketed reactor with $80 \mathrm{~g}$ of anthranilic acid as starting material according to the general procedure. After workup, crude product $(207 \mathrm{~g})$ was taken up in TBME $(160 \mathrm{~mL})$ and the mixture heated to $45{ }^{\circ} \mathrm{C}$ and then cooled to $0{ }^{\circ} \mathrm{C}$ for $30 \mathrm{~min}$. The resulting suspension was filtered off and the cake washed with TBME $(100 \mathrm{~mL})$. 
Pure quinazolinone 4a (86.5 g, 50\%) was obtained as a white crystalline solid.

3-Benzyl-5-methoxy-2-(trifluoromethyl)quinazolin-4-(3H)-one (4b). The reaction was performed according to the general procedure with anthranilic acid $\mathbf{1 b}$ as starting material, benzylamine and trifluoroacetic acid. The crude compound was purified by flash chromatography ( $n$-heptane/EtOAc: $100 / 0$ to $70 / 30$ ) yielding compound $\mathbf{4 b}$ as a yellow solid $(1.38 \mathrm{~g}, 69 \%)$. mp $117{ }^{\circ} \mathrm{C} .{ }^{1} \mathrm{H}$ NMR (500 MHz, DMSO) $\delta 7.87(\mathrm{t}, J=8.3 \mathrm{~Hz}, 1 \mathrm{H}), 7.39(\mathrm{~d}, J=8.0$ $\mathrm{Hz}, 1 \mathrm{H}), 7.24-7.34(\mathrm{~m}, 4 \mathrm{H}), 7.16(\mathrm{~d}, J=7.4 \mathrm{~Hz}, 2 \mathrm{H}), 5.27(\mathrm{~s}, 2 \mathrm{H})$, 3.89 (s, $3 \mathrm{H}) .{ }^{13} \mathrm{C}$ NMR (125 MHz, DMSO) $\delta 160.2,158.5,147.3$, $142.7(\mathrm{q}, J=34.8 \mathrm{~Hz}), 136.8,136.4,128.9,127.5,126.1,120.4,118.4$ $(\mathrm{q}, J=277.4 \mathrm{~Hz}), 111.9,111.3,56.7,47.3 .{ }^{19} \mathrm{~F}$ NMR $(375 \mathrm{MHz}$, DMSO) $\delta-64.99$. MS $[\mathrm{M}+\mathrm{H}]^{+} 335.16 \mathrm{~m} / z$. IR $1694(\mathrm{~s}), 1099(\mathrm{~s})$, $970(\mathrm{~s}), 817(\mathrm{~s}), 738(\mathrm{~s}) \mathrm{cm}^{-1}$. HRMS (ESI-TOF), $m / z[\mathrm{M}+\mathrm{H}]^{+}$ calcd for $\mathrm{C}_{17} \mathrm{H}_{14} \mathrm{~F}_{3} \mathrm{~N}_{2} \mathrm{O}_{2}$ 335.1002, found 335.1010.

3-Benzyl-7-methoxy-2-(trifluoromethyl)quinazolin-4-(3H)-one (4c). The reaction was performed according to the general procedure with anthranilic acid 1c as starting material, benzylamine and trifluoroacetic acid. The crude compound was purified by flash chromatography ( $n$-heptane/EtOAc: $100 / 0$ to $70 / 30$ ) yielding compound $4 \mathrm{c}$ as a yellow solid $(0.64 \mathrm{~g}, 65 \%$, contaminated with ca. $20 \mathrm{~mol} \%$ of $\mathrm{N}$-benzyl-2,2,2-trifluoroacetamide). mp $124^{\circ} \mathrm{C} .{ }^{1} \mathrm{H} \mathrm{NMR}$ $(500 \mathrm{MHz}, \mathrm{DMSO}) \delta 8.14(\mathrm{~d}, J=8.8 \mathrm{~Hz}, 1 \mathrm{H}), 7.24-7.38(\mathrm{~m}, 5 \mathrm{H})$, $7.16(\mathrm{~d}, J=7.2 \mathrm{~Hz}, 2 \mathrm{H}), 5.35(\mathrm{~s}, 2 \mathrm{H}), 3.96(\mathrm{~s}, 3 \mathrm{H}) .{ }^{13} \mathrm{C}$ NMR $(125$ MHz, DMSO) $\delta 165.2,147.3,142.93(\mathrm{q}, J=34.8 \mathrm{~Hz}), 137.9,136.7$, 128.9, 128.8, 127.8, 127.6, 126.1, 119.7, $115.8(\mathrm{q}, J=34.8 \mathrm{~Hz}), 110.0$, 56.7, 47.5. ${ }^{19} \mathrm{~F}$ NMR $\left(375 \mathrm{MHz}\right.$, DMSO) $\delta-64.74$. MS $[\mathrm{M}+\mathrm{H}]^{+}$ $335.16 \mathrm{~m} / z$. IR, 1680 (s), 1610 (s), 1106 (s), 1004 (s) $770(\mathrm{~s}) \mathrm{cm}^{-1}$. (ESI-TOF), $m / z[\mathrm{M}+\mathrm{H}]^{+}$calcd for $\mathrm{C}_{17} \mathrm{H}_{14} \mathrm{~N}_{2} \mathrm{O}_{2} \mathrm{~F}_{3}$ 335.1002, found 335.1020.

3-Benzyl-7-chloro-2-(trifluoromethyl)quinazolin-4-(3H)-one (4d). The reaction was performed according to the general procedure with anthranilic acid 1d as starting material, benzylamine and trifluoroacetic acid. The crude compound was purified by flash chromatography ( $n$ heptane/EtOAc: $100 / 0$ to $70 / 30$ ) yielding compound $4 \mathrm{~d}$ as an off white solid $(1.40 \mathrm{~g}, 72 \%) . \mathrm{mp} 105^{\circ} \mathrm{C} .{ }^{1} \mathrm{H}$ NMR (500 MHz, DMSO) $\delta$ $8.23(\mathrm{~d}, J=8.6 \mathrm{~Hz}, 1 \mathrm{H}), 8.03(\mathrm{~d}, J=1.9 \mathrm{~Hz}, 1 \mathrm{H}), 7.80\left(\mathrm{dd}, J_{1}=8.5\right.$ $\left.\mathrm{Hz}, J_{2}=2.0 \mathrm{~Hz}, 1 \mathrm{H}\right), 7.32(\mathrm{~m}, 2 \mathrm{H}), 7.19-7.27(\mathrm{~m}, 3 \mathrm{H}), 5.35(\mathrm{~s}, 2$ H). ${ }^{13} \mathrm{C}$ NMR (125 MHz, DMSO) $\delta 160.9,146.2,143.6$ (q, $J=35.4$ $\mathrm{Hz}), 140.5,136.3,130.5,129.2,128.9,128.0,127.6,126.2,121.1,118.5$ $(\mathrm{q}, J=277.5 \mathrm{~Hz}), 48.0 .{ }^{19} \mathrm{~F}$ NMR (375 MHz, DMSO) $\delta-64.83$. MS $[\mathrm{M}+\mathrm{H}]^{+} 339.00 \mathrm{~m} / z$. IR $1698(\mathrm{~s}), 1602$ (s), 1405 (s), 1199 (s), 750 (s) $\mathrm{cm}^{-1}$. (ESI-TOF), $m / z[\mathrm{M}+\mathrm{H}]^{+}$calcd for $\mathrm{C}_{16} \mathrm{H}_{11} \mathrm{ClF}_{3} \mathrm{~N}_{2} \mathrm{O}$ 339.0507 , found 339.0516

3-Benzyl-8-chloro-2-(trifluoromethyl)quinazolin-4-(3H)-one (4e). The reaction was performed according to the general procedure with anthranilic acid 1e as starting material, benzylamine and trifluoroacetic acid. The crude compound was purified by flash chromatography ( $n$ heptane/EtOAc: $100 / 0$ to $70 / 30$ ) yielding compound $4 \mathbf{e}$ as a yellow solid (0.98 g, 51\%). mp $87{ }^{\circ} \mathrm{C} .{ }^{1} \mathrm{H}$ NMR (500 MHz, DMSO) $\delta 8.19$ $\left(\mathrm{dd}, J_{1}=1.3 \mathrm{~Hz}, J_{2}=8.0 \mathrm{~Hz}, 1 \mathrm{H}\right), 8.15\left(\mathrm{dd}, J_{1}=1.4 \mathrm{~Hz}, J_{2}=7.9 \mathrm{~Hz}, 1\right.$ $\mathrm{H}), 7.73(\mathrm{t}, J=7.9 \mathrm{~Hz}, 1 \mathrm{H}), 7.22-7.34(\mathrm{~m}, 5 \mathrm{H}), 5.36(\mathrm{~s}, 2 \mathrm{H}) .{ }^{13} \mathrm{C}$ NMR (125 MHz, DMSO) $\delta 161.0,143.0(\mathrm{q}, J=35.6 \mathrm{~Hz}), 141.9$, $136.2,135.9,132.3,130.7,128.9,127.6,126.3,126.2,124.1,119.1$ (q, $J$ $=277.8 \mathrm{~Hz}), 48.2 .{ }^{19} \mathrm{~F}$ NMR $(375 \mathrm{MHz}, \mathrm{DMSO}) \delta-64.79$. MS $[\mathrm{M}+$ $\mathrm{H}^{+} 338.98 \mathrm{~m} / z$. IR 1680 (s), 1399 (s), 1205 (s), 988 (s), 765 (s). (ESI-TOF), $m / z[\mathrm{M}+\mathrm{H}]^{+}$calcd for $\mathrm{C}_{16} \mathrm{H}_{11} \mathrm{ClF}_{3} \mathrm{~N}_{2} \mathrm{O} 339.0507$, found 339.0520.

3-Benzyl-6-iodo-2-(trifluoromethyl)quinazolin-4-(3H)-one (4f). The reaction was performed according to the general procedure with anthranilic acid If as starting material, benzylamine and trifluoroacetic acid. The crude compound was purified by flash chromatography ( $n$-heptane/EtOAc: $100 / 0$ to $70 / 30$ ) yielding compound $4 \mathrm{f}$ as a yellow solid $(1.0 \mathrm{~g}, 63 \%)$. mp $105{ }^{\circ} \mathrm{C} .{ }^{1} \mathrm{H}$ NMR $(500 \mathrm{MHz}, \mathrm{DMSO}) \delta 8.50(\mathrm{~d}, J=2.0 \mathrm{~Hz}, 1 \mathrm{H}), 8.29\left(\mathrm{dd}, J_{1}=8.5 \mathrm{~Hz}\right.$, $\left.J_{2}=2.0 \mathrm{~Hz}, 1 \mathrm{H}\right), 7.69(\mathrm{~d}, J=8.5 \mathrm{~Hz}, 1 \mathrm{H}), 7.18-7.33(\mathrm{~m}, 5 \mathrm{H}), 5.35$ $(\mathrm{s}, 2 \mathrm{H}) .{ }^{13} \mathrm{C}$ NMR $(125 \mathrm{MHz}, \mathrm{DMSO}) \delta 160.1,144.4,144.3,142.8$ $(\mathrm{q}, J=35.7 \mathrm{~Hz}), 136.3,135.4,130.7,128.9,127.6,126.2,123.8,119.1$ $(\mathrm{q}, J=277.1 \mathrm{~Hz}), 96.3,48.1 .{ }^{19} \mathrm{~F}$ NMR (375 MHz, DMSO) $\delta-64.75$.
MS $[\mathrm{M}+\mathrm{H}]^{+} 430.98 \mathrm{~m} / z$. IR $1692(\mathrm{~s}), 1606(\mathrm{~m}), 1202(\mathrm{~s}), 843(\mathrm{~s})$, $722(\mathrm{~s}) \mathrm{cm}^{-1}$. (ESI-TOF), $m / z[\mathrm{M}+\mathrm{H}]^{+}$calcd for $\mathrm{C}_{16} \mathrm{H}_{11} \mathrm{~F}_{3} \mathrm{IN}_{2} \mathrm{O}$ 430.9862, found 430.9870 .

3-Benzyl-7-iodo-2-(trifluoromethyl)quinazolin-4-(3H)-one (4g). The reaction was performed according to the general procedure with anthranilic acid $\mathbf{1 g}$ as starting material, benzylamine and trifluoroacetic acid. The crude compound was purified by flash chromatography ( $n$-heptane/EtOAc: $100 / 0$ to $70 / 30$ ) yielding compound $4 \mathrm{~g}$ as a yellow solid $(1.09 \mathrm{~g}, 67 \%) . \mathrm{mp} 122{ }^{\circ} \mathrm{C} .{ }^{1} \mathrm{H}$ NMR $(500 \mathrm{MHz}, \mathrm{DMSO}) \delta 8.32(\mathrm{~d}, J=1.5 \mathrm{~Hz}, 1 \mathrm{H}), 8.08\left(\mathrm{dd}, J_{1}=\right.$ $\left.1.6 \mathrm{~Hz}, J_{2}=8.3 \mathrm{~Hz}, 1 \mathrm{H}\right), 7.95(\mathrm{~d}, J=8.3 \mathrm{~Hz}, 1 \mathrm{H}), 7.18-7.33(\mathrm{~m}, 5$ $\mathrm{H}), 5.34(\mathrm{~s}, 2 \mathrm{H}) .{ }^{13} \mathrm{C}$ NMR $(125 \mathrm{MHz}, \mathrm{DMSO}) \delta 161.2,145.8,143.2$ (q, $J=35.5 \mathrm{~Hz}), 138.9,137.1,136.3,128.9,128.6,127.6,126.2,121.6$, $117.9(\mathrm{q}, J=277.4 \mathrm{~Hz}), 104.0,47.9 .{ }^{19} \mathrm{~F}$ NMR (375 MHz, DMSO) $\delta$ -64.80 . MS $[\mathrm{M}+\mathrm{H}]^{+} 431.03 \mathrm{~m} / z$. IR $1693(\mathrm{~s}), 1588(\mathrm{~m}), 1199(\mathrm{~s})$, $998(\mathrm{~m}), 708(\mathrm{~s}) \mathrm{cm}^{-1}$. (ESI-TOF), $\mathrm{m} / z[\mathrm{M}+\mathrm{H}]^{+}$calcd for $\mathrm{C}_{16} \mathrm{H}_{11} \mathrm{~F}_{3} \mathrm{IN}_{2} \mathrm{O} 430.9862$, found 430.9879 .

3-Benzyl-6-fluoro-2-(trifluoromethyl)quinazolin-4-(3H)-one (4h). The reaction was performed according to the general procedure with anthranilic acid $1 \mathrm{~h}$ as starting material, benzylamine and trifluoroacetic acid. The crude compound was purified by flash chromatography ( $n$ heptane/EtOAc: $100 / 0$ to 50/50) yielding compound $4 \mathrm{~h}$ as a white solid (0.59 g, 59\%). mp $94{ }^{\circ} \mathrm{C} .{ }^{1} \mathrm{H}$ NMR (500 MHz, DMSO) $\delta 8.01$ $\left(\mathrm{dd}, J_{1}=8.9 \mathrm{~Hz}, J_{2}=4.9 \mathrm{~Hz}, 1 \mathrm{H}\right), 7.87-7.95(\mathrm{~m}, 2 \mathrm{H}), 7.19-7.33(\mathrm{~m}$, $5 \mathrm{H}), 5.36(\mathrm{~s}, 2 \mathrm{H}) .{ }^{13} \mathrm{C}$ NMR (125 MHz, DMSO) $\delta 163.3,161.3$, $160.8(\mathrm{~d}, J=3 \mathrm{~Hz}), 142.0,136.3,131.9(\mathrm{~d}, J=9 \mathrm{~Hz}), 128.9,127.6$, $126.2,124.4(\mathrm{~d}, J=24.3 \mathrm{~Hz}), 123.9(\mathrm{~d}, J=9.1 \mathrm{~Hz}), 118.7(\mathrm{q}, J=277.1$ $\mathrm{Hz}), 112.2(\mathrm{~d}, J=24.1 \mathrm{~Hz}), 48.0 .{ }^{19} \mathrm{~F}$ NMR $(375 \mathrm{MHz}, \mathrm{DMSO}) \delta$ $-64.63,-109.01$. MS $[\mathrm{M}+\mathrm{H}]^{+} 323.13 \mathrm{~m} / z$. IR 1694 (s), 1202 (s), $992(\mathrm{~m}), 726(\mathrm{~s}) \mathrm{cm}^{-1}$. (ESI-TOF), $\mathrm{m} / z[\mathrm{M}+\mathrm{H}]^{+}$calcd for $\mathrm{C}_{16} \mathrm{H}_{11} \mathrm{~F}_{4} \mathrm{~N}_{2} \mathrm{O}$ 323.0802, found 323.0813.

3-Benzyl-2,7-bis(trifluoromethyl)quinazolin-4-(3H)-one (4i). The reaction was performed according to the general procedure with anthranilic acid $\mathbf{l i}$ as starting material, benzylamine and trifluoroacetic acid. The crude compound was purified by flash chromatography $(n-$ heptane/EtOAc: $100 / 0$ to $70 / 30$ ) yielding compound $4 \mathbf{i}$ as a yellow thick oil (1.28 g, 72\%). ${ }^{1} \mathrm{H}$ NMR (500 MHz, DMSO) $\delta 8.43(\mathrm{~d}, J=$ $8.3 \mathrm{~Hz}, 1 \mathrm{H}), 8.29(\mathrm{~s}, 1 \mathrm{H}), 8.06\left(\mathrm{dd}, J_{1}=8.3 \mathrm{~Hz}, J_{2}=1.3 \mathrm{~Hz}, 1 \mathrm{H}\right)$, $7.18-7.34(\mathrm{~m}, 5 \mathrm{H}), 5.38(\mathrm{~s}, 2 \mathrm{H}){ }^{13} \mathrm{C}$ NMR $(125 \mathrm{MHz}, \mathrm{DMSO}) \delta$ $160.9,145.3,143.8(\mathrm{q}, J=35.5 \mathrm{~Hz}), 135.3(\mathrm{q}, J=32.5 \mathrm{~Hz}), 129.0$, 128.9, 127.6, 126.2, $126.0(\mathrm{~m}), 125.2,124.9,123.8(\mathrm{q}, J=273.2 \mathrm{~Hz})$, $118.46(\mathrm{q}, J=277.5 \mathrm{~Hz}), 55.4,48.2 .{ }^{19} \mathrm{~F}$ NMR $(375 \mathrm{MHz}, \mathrm{DMSO}) \delta$ -61.69, -64.87. MS $[\mathrm{M}+\mathrm{H}]^{+} 373.12 \mathrm{~m} / z$. IR $1701(\mathrm{~s}), 1609(\mathrm{~s})$, $1316(\mathrm{~s}), 1205(\mathrm{~s}), 973(\mathrm{~m}), 692(\mathrm{~s}) \mathrm{cm}^{-1}$. (ESI-TOF), $m / z[\mathrm{M}+\mathrm{H}]^{+}$ calcd for $\mathrm{C}_{17} \mathrm{H}_{11} \mathrm{~F}_{6} \mathrm{~N}_{2} \mathrm{O}$ 373.0770, found 373.0775.

General Procedure for 3-Benzyl-2-(trifluoromethyl)quinazolin-4(3H)-ones $4 \mathbf{j}-\mathrm{I}, \mathbf{0}$ (Strategy II). A prepared solution of the trifluoroacetic acid (1.0 equiv) in T3P $(50 \% \mathrm{w} / \mathrm{w}$ in toluene, 1.0 equiv) was added at $\mathrm{rt}$ dropwise to a suspension of the corresponding anthranilic acids $\mathbf{1 j}-\mathbf{l}, \mathbf{o}(1 \mathrm{~g}, 1.0$ equiv) in toluene $(4 \mathrm{~mL})$. After stirring until total consumption of $\mathbf{1 j}-\mathbf{l}, \mathbf{o}$ (checked by LC-MS, $3-5 \mathrm{~h}$ typically), $\mathrm{Et}_{3} \mathrm{~N}$ (1.0 equiv) and $\mathrm{T} 3 \mathrm{P}(50 \% \mathrm{w} / \mathrm{w}$ in toluene, 1.0 equiv) were sequentially added followed by benzylamine ( 0.98 equiv). The resulting reaction mixture was heated to $70{ }^{\circ} \mathrm{C}$ and kept it for $14 \mathrm{~h}$ followed by $5-7 \mathrm{~h}$ at reflux to achieve complete cyclization. The cooled reaction mixture was quenched by dropwise addition of aq. sat. $\mathrm{NaHCO}_{3}(25 \mathrm{~mL}$, Caution! Gas evolution) followed by extraction with solvents such as EtOAc/iPrOAc or DCM $(1 \times 25 \mathrm{~mL})$. Collected organic phases were washed with $25 \mathrm{~mL}$ of aq. sat. $\mathrm{NaHCO}_{3}$ and brine $(25 \mathrm{~mL})$ respectively, dried over anhydrous $\mathrm{MgSO}_{4}$, filtered and evaporated under reduced pressure to afford desired crude quinazolino-4-ones $4 \mathbf{j}-\mathbf{l}, \mathbf{o}$.

3-Benzyl-7-nitro-2-(trifluoromethyl)quinazolin-4-(3H)-one (4j). The reaction was performed according to the general procedure with anthranilic acid $\mathbf{1 j}$ as starting material, benzylamine and trifluoroacetic acid. The crude compound was purified by flash chromatography ( $n$-heptane/EtOAc: $100 / 0$ to $70 / 30$ ) yielding compound $4 \mathbf{j}$ as a light yellow solid $(1.43 \mathrm{~g}, 75 \%)$ mp $110^{\circ} \mathrm{C} .{ }^{1} \mathrm{H}$ NMR (500 MHz, DMSO) $\delta 8.62(\mathrm{t}, J=1.1 \mathrm{~Hz}, 1 \mathrm{H}), 8.46(\mathrm{~d}, J=1.0$ $\mathrm{Hz}, 2 \mathrm{H}), 7.24-7.36(\mathrm{~m}, 5 \mathrm{H}), 5.37(\mathrm{~s}, 2 \mathrm{H}) .{ }^{13} \mathrm{C}$ NMR $(125 \mathrm{MHz}$, 
DMSO) $\delta 160.7,152.1,145.6,144.3(\mathrm{q}, J=35.6 \mathrm{~Hz}), 138.0,136.0$, $129.5,128.9,127.8,126.6,126.3,123.8,118.8(\mathrm{q}, J=277.8 \mathrm{~Hz}), 48.4$. ${ }^{19} \mathrm{~F}$ NMR (375 MHz, DMSO) $\delta-64.90$. MS $[\mathrm{M}+\mathrm{H}]^{+}+\mathrm{NH}_{4}^{+}$ $366.99 \mathrm{~m} / z$. IR 1698 (s), 1529 (s), 1404 (s), 1199 (s), 982 (s), 687 (s) $\mathrm{cm}^{-1}$. (ESI-TOF), $m / z[\mathrm{M}+\mathrm{H}]^{+}$calcd for $\mathrm{C}_{16} \mathrm{H}_{11} \mathrm{~F}_{3} \mathrm{~N}_{3} \mathrm{O}_{3} 350.0747$, found 350.0746 .

3-Benzyl-2-(trifluoromethyl)benzoquinazolin-4-(3H)-one (4k). The reaction was performed according to the general procedure with anthranilic acid $\mathbf{1 k}$ as starting material, benzylamine and trifluoroacetic acid. The crude compound was purified by flash chromatography ( $n$-heptane/EtOAc: $100 / 0$ to $70 / 30$ ) yielding compound $4 \mathbf{k}$ as a white solid (1.04 g, 55\%). mp $129{ }^{\circ} \mathrm{C} .{ }^{1} \mathrm{H}$ NMR $(500 \mathrm{MHz}, \mathrm{DMSO}) \delta 8.97(\mathrm{~s}, 1 \mathrm{H}), 8.56(\mathrm{~s}, 1 \mathrm{H}), 8.33(\mathrm{~d}, J=8.2 \mathrm{~Hz}$ $1 \mathrm{H}), 8.23(\mathrm{~d}, J=8.2 \mathrm{~Hz}, 1 \mathrm{H}), 7.75(\mathrm{~m}, 2 \mathrm{H}), 7.21-7.34(\mathrm{~m}, 5 \mathrm{H})$, $5.40(\mathrm{~s}, 2 \mathrm{H}) .{ }^{13} \mathrm{C}$ NMR $(125 \mathrm{MHz}, \mathrm{DMSO}) \delta 162.0,141.5(\mathrm{q}, J=$ $35.1 \mathrm{~Hz}), 140.3,136.8,136.6,132.8,129.9,129.7,128.9,128.8,128.2$, $127.5,127.5,126.1,118.7(\mathrm{q}, J=277.1 \mathrm{~Hz}), 47.6 .{ }^{19} \mathrm{~F}$ NMR $(375$ $\mathrm{MHz}, \mathrm{DMSO}) \delta-64.60$. MS $[\mathrm{M}+\mathrm{H}]^{+} 355.13 \mathrm{~m} / z$. IR 1693 (s), 1609 (s), $1186(\mathrm{~s}), 905(\mathrm{~s}), 698(\mathrm{~s}) \mathrm{cm}^{-1}$. (ESI-TOF), $\mathrm{m} / z[\mathrm{M}+\mathrm{H}]^{+}$calcd for $\mathrm{C}_{20} \mathrm{H}_{14} \mathrm{~F}_{3} \mathrm{~N}_{2} \mathrm{O}$ 355.1053, found 355.1061.

3-Benzyl-2-(trifluoromethyl)pteridin-4(3H)-one (4I). The reaction was performed according to the general procedure with anthranilic acid $\mathbf{1 l}$ as starting material, benzylamine and trifluoroacetic acid. The crude compound was purified by flash chromatography (DCM/ $\mathrm{MeOH}: 100 / 0$ to $90 / 10)$ yielding compound 41 as a yellow foam (1.05 g, 48\%). ${ }^{1} \mathrm{H}$ NMR (400 MHz, DMSO) $\delta 9.18(\mathrm{~d}, J=2.1 \mathrm{~Hz}, 1 \mathrm{H})$, $9.05(\mathrm{~d}, J=2.1 \mathrm{~Hz}, 1 \mathrm{H}), 7.27-7.35(\mathrm{~m}, 5 \mathrm{H}), 5.38(\mathrm{~s}, 2 \mathrm{H}) .{ }^{13} \mathrm{C} \mathrm{NMR}$ $(125 \mathrm{MHz}, \mathrm{DMSO}) \delta 160.9,152.1,151.4,147.3,145.6(\mathrm{q}, J=36 \mathrm{~Hz})$, $135.9,134.7,128.8,127.6,126.3,118.4(\mathrm{q}, J=277.9 \mathrm{~Hz}), 48.6(\mathrm{~d}, J=3$ $\mathrm{Hz}) .{ }^{19} \mathrm{~F}$ NMR (375 MHz, DMSO) $\delta-65.11$. MS $[\mathrm{M}+\mathrm{H}]^{+} 307.18$ $\mathrm{m} / z$. IR $1728(\mathrm{~m}), 1347(\mathrm{~s}), 1120(\mathrm{~s}), 1125(\mathrm{~s}), 731(\mathrm{~s}), 695(\mathrm{~s}) \mathrm{cm}^{-1}$. (ESI-TOF), $m / z[\mathrm{M}+\mathrm{H}]^{+}$calcd for $\mathrm{C}_{14} \mathrm{H}_{10} \mathrm{~F}_{3} \mathrm{~N}_{4} \mathrm{O}[\mathrm{M}+\mathrm{H}]^{+}$ 307.0801 , found 307.0816 .

3-Benzyl-2-(trifluoromethyl)pyrido[2,3-d]pyrimidin-4(3H)-one $(4 \mathrm{~m})$. The reaction was performed according to the general procedure with anthranilic acid $\mathbf{1 m}$ as starting material, benzylamine and trifluoroacetic acid. The crude compound was purified by flash chromatography ( $n$-heptane/EtOAc: $100 / 0$ to $70 / 30$ ) yielding compound $4 \mathrm{~m}$ as a light yellow solid $(0.55 \mathrm{~g}, 25 \%)$. mp $127^{\circ} \mathrm{C} .{ }^{1} \mathrm{H}$ NMR $(500 \mathrm{MHz}, \mathrm{DMSO}) \delta 9.13\left(\mathrm{dd}, J_{1}=4.5 \mathrm{~Hz}, J_{2}=1.9 \mathrm{~Hz}, 1 \mathrm{H}\right)$, $8.63\left(\mathrm{dd}, J_{1}=7.9 \mathrm{~Hz}, J_{2}=1.9 \mathrm{~Hz}, 1 \mathrm{H}\right), 7.77\left(\mathrm{dd}, J_{1}=7.9 \mathrm{~Hz}, J_{2}=4.6\right.$ $\mathrm{Hz}, 1 \mathrm{H}), 7.23-7.34(\mathrm{~m}, 5 \mathrm{H}), 5.36(\mathrm{~s}, 2 \mathrm{H}) .{ }^{13} \mathrm{C} \mathrm{NMR}(125 \mathrm{MHz}$, DMSO) $\delta 162.1,157.2,155.5,145.3(\mathrm{q}, J=35.9 \mathrm{~Hz}), 136.9,136.2$, $128.9,127.6,126.2,125.5,121.7,120.3(\mathrm{q}, J=277.4 \mathrm{~Hz}), 48.2 .{ }^{19} \mathrm{~F}$ NMR (375 MHz, DMSO) $\delta-65.03$. MS $[\mathrm{M}+\mathrm{H}]^{+} 305.88 \mathrm{~m} / z$. IR 1698 (s), 1394 (s), 1211 (s), $731(\mathrm{~s}) \mathrm{cm}^{-1}$. (ESI-TOF), $m / z[\mathrm{M}+\mathrm{H}]^{+}$ calcd for $\mathrm{C}_{15} \mathrm{H}_{11} \mathrm{~F}_{3} \mathrm{~N}_{3} \mathrm{O} 306.0849$, found 306.0852.

3-Benzyl-6-bromo-2-(trifluoromethyl)pyrido[2,3-d]pyrimidin$4(3 \mathrm{H})$-one (4n). The reaction was performed according to the general procedure with anthranilic acid $\mathbf{1 n}$ as starting material, benzylamine and trifluoroacetic acid. The crude compound was purified by flash chromatography ( $n$-heptane/EtOAc: $100 / 0$ to $70 / 30$ ) yielding compound $4 \mathrm{n}$ as a light yellow thick oil $(0.37 \mathrm{~g}, 21 \%) .{ }^{1} \mathrm{H}$ NMR $(500 \mathrm{MHz}, \mathrm{DMSO}) \delta 9.24(\mathrm{~d}, J=2.6 \mathrm{~Hz}, 1 \mathrm{H}), 8.79(\mathrm{~d}, J=2.6 \mathrm{~Hz}, 1$ $\mathrm{H}), 7.25-7.33(\mathrm{~m}, 5 \mathrm{H}), 5.35$ (s, $2 \mathrm{H}) .{ }^{13} \mathrm{C}$ NMR (125 MHz, DMSO) $\delta 161.3,157.9,154.2,145.5(\mathrm{q}, J=35.8 \mathrm{~Hz}), 138.4,135.9,129.0$, $128.9,127.8,127.7,126.3,119.1(\mathrm{q}, J=276.80 \mathrm{~Hz}), 48.4 .{ }^{19} \mathrm{~F}$ NMR $(375 \mathrm{MHz}, \mathrm{DMSO}) \delta-65.08$. MS $[\mathrm{M}+\mathrm{H}]^{+} 385.75 \mathrm{~m} / z$. IR $1696(\mathrm{~s})$, 1449 (s), 1308 (s), $1210(\mathrm{~s}), 808(\mathrm{~s}), 729,(\mathrm{~s}), 695(\mathrm{~s}) \mathrm{cm}^{-1}$. (ESITOF), $m / z[\mathrm{M}+\mathrm{H}]^{+}$calcd for $\mathrm{C}_{15} \mathrm{H}_{11} \mathrm{BrF}_{3} \mathrm{~N}_{3} \mathrm{O}$ 383.9954, found 383.9958.

3-Benzyl-2-(trifluoromethyl)pyrido[3,4-d]pyrimidin-4(3H)-one (40). The reaction was performed according to the general procedure with anthranilic acid 1o as starting material, benzylamine and trifluoroacetic acid. The crude compound was purified by flash chromatography ( $n$-heptane/EtOAc: from $100 / 0$ to $50 / 50$ ) yielding compound $4 \mathrm{o}$ as a light yellow solid $(0.83 \mathrm{~g}, 38 \%)$. mp $106{ }^{\circ} \mathrm{C} .{ }^{1} \mathrm{H}$ NMR (500 MHz, DMSO) $\delta 9.29(\mathrm{~s}, 1 \mathrm{H}), 8.88(\mathrm{~d}, J=5.2 \mathrm{~Hz}, 1 \mathrm{H})$, $8.09(\mathrm{~d}, J=5.2 \mathrm{~Hz}, 1 \mathrm{H}), 7.23-7.33(\mathrm{~m}, 5 \mathrm{H}), 5.36(\mathrm{~s}, 2 \mathrm{H}) .{ }^{13} \mathrm{C} \mathrm{NMR}$ $(125 \mathrm{MHz}, \mathrm{DMSO}) \delta 160.8,151.7,149.4,144.1(\mathrm{q}, J=35.7 \mathrm{~Hz})$, $140.0,136.0,128.9,127.7,127.6,126.3,119.4(\mathrm{q}, J=277.6 \mathrm{~Hz}), 119.2$, 48.4. ${ }^{19} \mathrm{~F}$ NMR (375 MHz, DMSO) $\delta-64.81$. MS $[\mathrm{M}+\mathrm{H}]^{+} 305.92$ $m / z$. IR $1703(\mathrm{~s}), 1401$ (s), $1202(\mathrm{~s}), 693(\mathrm{~s}) \mathrm{cm}^{-1}$. (ESI-TOF), $\mathrm{m} / z$ $[\mathrm{M}+\mathrm{H}]^{+}$calcd for $\mathrm{C}_{15} \mathrm{H}_{11} \mathrm{~F}_{3} \mathrm{~N}_{3} \mathrm{O} 306.0849$, found 306.0852

3-(o-Tolyl)-2-(trifluoromethyl)quinazolin-4-(3H)-one (4r). The reaction was performed according to the general procedure with anthranilic acid $\mathbf{1 r}$ as starting material, $o$-toluidine and trifluoroacetic acid. The crude compound was purified by flash chromatography ( $n$ heptane/EtOAc: from $100 / 0$ to $70 / 30$ ) yielding compound $\mathbf{4 r}$ as a white solid (0.92 g, 42\%). mp $144{ }^{\circ} \mathrm{C} .{ }^{1} \mathrm{H}$ NMR $\left(500 \mathrm{MHz}, \mathrm{CDCl}_{3}\right) \delta$ 8.38-8.40 (m, $1 \mathrm{H}), 7.91-7.96(\mathrm{~m}, 2 \mathrm{H}), 7.69(\mathrm{~m}, 1 \mathrm{H}), 7.36-7.48$ $(\mathrm{m}, 3 \mathrm{H}), 7.23(\mathrm{~d}, J=7.8 \mathrm{~Hz}, 1 \mathrm{H}), 2.16(\mathrm{~s}, 3 \mathrm{H}) .{ }^{13} \mathrm{C}$ NMR $(125$ $\left.\mathrm{MHz}_{\mathrm{CDCl}}\right) \delta 161.0,145.4,142.4(\mathrm{q}, J=35.5 \mathrm{~Hz}), 136.9,135.3$, $133.9,131.0,130.3,129.6,129.1,128.8,127.5,126.9,122.2,117.8$ (q, $J$ $=277.5 \mathrm{~Hz}), 17.51 .{ }^{19} \mathrm{~F}$ NMR $\left(375 \mathrm{MHz}, \mathrm{CDCl}_{3}\right) \delta-65.58$. MS $[\mathrm{M}+$ $\mathrm{H}]^{+} 305.21 \mathrm{~m} / z$. IR $1692(\mathrm{~s}), 1375$ (s), 1202 (s), 965 (s), 693 (s) $\mathrm{cm}^{-1}$. (ESI-TOF), $m / z[\mathrm{M}+\mathrm{H}]^{+}$calcd for $\mathrm{C}_{16} \mathrm{H}_{12} \mathrm{~F}_{3} \mathrm{~N}_{2} \mathrm{O}$ 305.0896, found 305.0898 .

3-Pentyl-2-(trifluoromethyl)quinazolin-4(3H)-one (4s). The reaction was performed according to the general procedure with anthranilic acid $1 \mathrm{~s}$ as starting material, $n$-pentylamine and trifluoroacetic acid. The crude compound was purified by flash chromatography ( $n$-heptane/EtOAc: from $100 / 0$ to $70 / 30$ ) yielding compound $4 \mathrm{~s}$ as a transparent oil $(1.23 \mathrm{~g}, 59 \%) .{ }^{1} \mathrm{H}$ NMR $(500 \mathrm{MHz}, \mathrm{DMSO}) \delta$ $8.23(\mathrm{~d}, J=7.7 \mathrm{~Hz}, 1 \mathrm{H}), 7.94-7.97(\mathrm{~m}, 1 \mathrm{H}), 7.85(\mathrm{~d}, J=8.1 \mathrm{~Hz}, 1$ H), $7.73(\mathrm{t}, J=7.7 \mathrm{~Hz}, 1 \mathrm{H}), 4.01(\mathrm{t}, J=8.0 \mathrm{~Hz}, 2 \mathrm{H}), 1.67-1.72(\mathrm{~m}, 2$ $\mathrm{H}), 1.35(\mathrm{~m}, 4 \mathrm{H}), 0.88-0.91(\mathrm{~m}, 3 \mathrm{H}) .{ }^{13} \mathrm{C}$ NMR $(125 \mathrm{MHz}$, DMSO) $\delta 161.1,145.0,142.1(\mathrm{q}, J=34.9 \mathrm{~Hz}), 135.7,130.0,128.6$, $127.0,122.1,118.7(\mathrm{q}, J=276.8 \mathrm{~Hz}), 45.4,28.9,28.0,22.1,14.3 .{ }^{19} \mathrm{~F}$ NMR (375 MHz, DMSO) $\delta-64.88$. MS $[\mathrm{M}+\mathrm{H}]^{+}$285.26. IR 2933 (m), 1690 (s), 1609 (s), 1467 (s), 1404 (s), 1321 (s), 1238 (s), 1202 (s), $1092(\mathrm{~s}), 772(\mathrm{~s}), 695(\mathrm{~s}) \mathrm{cm}^{-1}$. (ESI-TOF), $m / z[\mathrm{M}+\mathrm{H}]^{+}$calcd for $\mathrm{C}_{14} \mathrm{H}_{16} \mathrm{~F}_{3} \mathrm{~N}_{2} \mathrm{O}$ 285.1209, found 285.1216 .

(S)-3-Isobutyl-3,4-dihydro-1H-benzo[e][1,4]diazepine-2,5-dione (6). ${ }^{15 \mathrm{C}}$ To a suspension of anthranilic acid 1a $(1.0 \mathrm{~g}, 7.22 \mathrm{mmol}, 1.0$ equiv) in toluene $(4 \mathrm{~mL})$ at $\mathrm{rt}$ was added $\mathrm{Et}_{3} \mathrm{~N}(1.5 \mathrm{~mL}, 10.8 \mathrm{mmol}$, 1.5 equiv) followed by T3P ( $50 \% \mathrm{w} / \mathrm{w}$ in toluene, $4.38 \mathrm{~mL}, 7.22$ mmol, 1.0 equiv). The resulting mixture was kept at $\mathrm{rt}$ for $2.5 \mathrm{~h}$ then heated to $70^{\circ} \mathrm{C}$ and L-leucine methyl ester hydrochloride $(1.3 \mathrm{~g}, 7.07$ mmol, 0.98 equiv) added in one portion. After $20 \mathrm{~h}$ at $70^{\circ} \mathrm{C}$, the reaction mixture was cooled to rt, diluted with EtOAc $(25 \mathrm{~mL})$ and quenched by slow addition of aq. sat. $\mathrm{NaHCO}_{3}(25 \mathrm{~mL}$, Caution! Gas evolution). Aqueous phase was extracted with EtOAc $(25 \mathrm{~mL})$ and collected organic phases were washed with aq. sat. $\mathrm{NaHCO}_{3}(25 \mathrm{~mL})$ and brine $(25 \mathrm{~mL})$, dried over anhydrous $\mathrm{MgSO}_{4}$, filtered and evaporated under reduced pressure to afford desired crude 6 . After trituration with TBME $(10 \mathrm{~mL})$, purified benzodiazepine dione 6 was obtained as a white powder $(0.53 \mathrm{~g}, 32 \%) . \mathrm{mp} 242{ }^{\circ} \mathrm{C} .{ }^{1} \mathrm{H}$ NMR $(500$ MHz, DMSO) $\delta 10.38(\mathrm{~s}, 1 \mathrm{H}), 8.45(\mathrm{~d}, J=5.8 \mathrm{~Hz}, 1 \mathrm{H}), 7.74\left(\mathrm{dd}, J_{1}\right.$ $\left.=1.5 \mathrm{~Hz}, J_{2}=7.8 \mathrm{~Hz}, 1 \mathrm{H}\right), 7.52(\mathrm{~m}, 1 \mathrm{H}), 7.21-7.24(\mathrm{~m}, 1 \mathrm{H}), 7.10$ $(\mathrm{d}, J=8.0 \mathrm{~Hz}, 1 \mathrm{H}), 3.61(\mathrm{q}, J=7.2 \mathrm{~Hz}, 1 \mathrm{H}), 1.66-1.75(\mathrm{~m}, 1 \mathrm{H})$, $1.56(\mathrm{t}, J=7.2 \mathrm{~Hz}, 2 \mathrm{H}), 0.86(\mathrm{~d}, J=6.6 \mathrm{~Hz}, 3 \mathrm{H}), 0.78(\mathrm{~d}, J=6.6 \mathrm{~Hz}$, $3 \mathrm{H}) .{ }^{13} \mathrm{C}$ NMR (125 MHz, DMSO) $\delta 172.1,168.2,137.2,132.7$, $130.8,126.8,124.4,121.4,36.6,24.3,23.3,22.0$. MS $[\mathrm{M}+\mathrm{H}]^{+} 233.20$. The analytical data are consistent with reported data.

3-Benzyl-2-(chlorodifluoromethyl)quinazolin-4-(3H)-one (4t). The reaction was performed according to the general procedure with anthranilic acid 1t as starting material, benzylamine and chlorodifluoroacetic acid. The crude compound was purified by flash chromatography ( $n$-heptane/EtOAc: from $100 / 0$ to $80 / 20$ ) yielding compound $4 \mathrm{t}$ as a transparent oil $(1.13 \mathrm{~g}, 49 \%) .{ }^{1} \mathrm{H}$ NMR $(500 \mathrm{MHz}$, $\left.\mathrm{CDCl}_{3}\right) \delta 8.36\left(\mathrm{dt}, J_{1}=1.0 \mathrm{~Hz}, J_{2}=8.0 \mathrm{~Hz}, 1 \mathrm{H}\right), 7.88-7.89(\mathrm{~m}, 2 \mathrm{H})$, 7.63-7.67 (m, $1 \mathrm{H}), 7.26-7.35(\mathrm{~m}, 3 \mathrm{H}), 7.17(\mathrm{~d}, J=7.3 \mathrm{~Hz}, 2 \mathrm{H})$, $5.57(\mathrm{~s}, 2 \mathrm{H}) .{ }^{13} \mathrm{C}$ NMR $\left(125 \mathrm{MHz}, \mathrm{CDCl}_{3}\right) \delta 161.8,145.8,145.6(\mathrm{t}, J$ $=28.7 \mathrm{~Hz}), 145.0,135.6,135.1,129.3,128.7,127.5,127.4,126.1$, 121.6, $119.5(\mathrm{t}, J=294.3 \mathrm{~Hz}), 48.3 .{ }^{19} \mathrm{~F}$ NMR $\left(375 \mathrm{MHz}, \mathrm{CDCl}_{3}\right) \delta$ -53.79. MS $[\mathrm{M}+\mathrm{H}]^{+}$321.16. IR $1690(\mathrm{~s}), 1602(\mathrm{~s}), 1167$ (s), 1114 (s), $924(\mathrm{~s}), 809(\mathrm{~m}), 772(\mathrm{~s}) \mathrm{cm}^{-1}$. (ESI-TOF), $m / z[\mathrm{M}+\mathrm{H}]^{+}$calcd for $\mathrm{C}_{16} \mathrm{H}_{12} \mathrm{ClF}_{2} \mathrm{~N}_{2} \mathrm{O}$ 321.0601, found 321.0603 . 
3-Benzyl-2-(perfluoroethyl)quinazolin-4-(3H)-one (4u). The reaction was performed according to the general procedure with anthranilic acid $\mathbf{1} \mathbf{u}$ as starting material, benzylamine and trifluoroacetic acid. The crude compound was purified by flash chromatography ( $n$ heptane/EtOAc: from $100 / 0$ to $70 / 30$ ) yielding compound $4 \mathbf{u}$ as a light brown solid (1.18 g, 46\%). mp $62{ }^{\circ} \mathrm{C}$. ${ }^{1} \mathrm{H}$ NMR $(500 \mathrm{MHz}$, $\left.\mathrm{CDCl}_{3}\right) \delta 8.36-8.38(\mathrm{~m}, 1 \mathrm{H}), 7.87(\mathrm{~m}, 2 \mathrm{H}), 7.66(\mathrm{~m}, 1 \mathrm{H}), 7.30-$ $7.36(\mathrm{~m}, 3 \mathrm{H}), 7.21(\mathrm{~d}, J=7.3 \mathrm{~Hz}, 2 \mathrm{H}), 5.51(\mathrm{~s}, 2 \mathrm{H}) .{ }^{13} \mathrm{C}$ NMR $(125$ $\left.\mathrm{MHz}, \mathrm{CDCl}_{3}\right) \delta 161.6,144.8,142.1(\mathrm{t}, J=27.6 \mathrm{~Hz}), 135.7,135.0$, 129.7, 128.7, 127.6, 127.3, 126.3, 122.0, $119.5(\mathrm{t}, J=34.4 \mathrm{~Hz}), 117.2$ $(\mathrm{q}, J=34.2 \mathrm{~Hz}), 110.9(\mathrm{~m}), 47.6 .{ }^{19} \mathrm{~F}$ NMR $\left(375 \mathrm{MHz}, \mathrm{CDCl}_{3}\right) \delta$ $-108.99,-79.62$. MS $[\mathrm{M}+\mathrm{H}]^{+} 355.09 \mathrm{~m} / z$. IR $1686(\mathrm{~s}), 1608(\mathrm{~s})$, 1397 (s), 1234 (s), 1194 (s), 1134 (s), 1072 (s), 944 (s723 (s) cm $\mathrm{cm}^{-1}$. (ESI-TOF), $m / z[\mathrm{M}+\mathrm{H}]^{+}$calcd for $\mathrm{C}_{17} \mathrm{H}_{12} \mathrm{~F}_{5} \mathrm{~N}_{2} \mathrm{O}$ 355.0864, found 355.0870 .

1-Methyl-3-propyl-5-(trifluoromethyl)-1,6-dihydro-7H-pyrazolo[4,3-d]pyrimidin-7-one (8). A prepared solution of the trifluoroacetic acid $(0.42 \mathrm{~mL}, 5.38 \mathrm{mmol}, 1.0$ equiv) in T3P $(50 \% \mathrm{w} / \mathrm{w}$ in toluene, 3.3 $\mathrm{mL}, 5.38 \mathrm{mmol}, 1.0$ equiv) was added dropwise to a suspension 4amino-1-methyl-3- $\mathrm{N}$-propyl-1H-pyrazole-5-carboxamide (7) (1.0 g, $5.38 \mathrm{mmol}, 1.0$ equiv) in toluene $(4 \mathrm{~mL})$ at $\mathrm{rt}$. After $4 \mathrm{~h}$ the mixture was heated to reflux for $1 \mathrm{~h}$ to complete the cyclization. The cooled reaction mixture was diluted with EtOAc $(45 \mathrm{~mL})$, quenched by dropwise addition of aq. sat. $\mathrm{NaHCO}_{3}(25 \mathrm{~mL}$, Caution! Gas evolution) followed by extraction with EtOAc $(25 \mathrm{~mL})$. Collected organic phases were washed with $25 \mathrm{~mL}$ of aq. sat. $\mathrm{NaHCO}_{3}$ and brine $(25 \mathrm{~mL})$ respectively, dried over anhydrous $\mathrm{MgSO}_{4}$, filtered and evaporated under reduced pressure to afford the crude compound as a yellow-white powder which was purified by trituration with EtOAc $(20$ $\mathrm{mL})$ to yield 8 as a white powder $(0.85 \mathrm{~g}, 61 \%) . \mathrm{mp} 202{ }^{\circ} \mathrm{C} .{ }^{1} \mathrm{H}$ NMR $\left(500 \mathrm{MHz}, \mathrm{CDCl}_{3}\right) \delta 10.8(\mathrm{br}, \mathrm{s}, 1 \mathrm{H}), 4.31(\mathrm{~s}, 3 \mathrm{H}), 2.93(\mathrm{t}, J=7.5$ $\mathrm{Hz}, 2 \mathrm{H}), 1.84(\mathrm{~m}, 2 \mathrm{H}), 1.03(\mathrm{t}, J=7.4 \mathrm{~Hz}, 3 \mathrm{H}) .{ }^{13} \mathrm{C}$ NMR $(125$ $\left.\mathrm{MHz}, \mathrm{CDCl}_{3}\right) \delta 153.8,148.0,139.0(\mathrm{q}, J=39.1 \mathrm{~Hz}), 136.5,125.4$, $118.1(\mathrm{q}, J=275.5 \mathrm{~Hz}), 38.5,27.5,22.3,13.9 .{ }^{19} \mathrm{~F}$ NMR $(375 \mathrm{MHz}$, $\left.\mathrm{CDCl}_{3}\right) \delta-69.37$. MS $[\mathrm{M}+\mathrm{H}]^{+} 261.30 \mathrm{~m} / z$. IR $1690(\mathrm{~s}), 1321(\mathrm{~s})$, 1217 (s), 877 (s), 760(s), $706(\mathrm{~s}) \mathrm{cm}^{-1}$. (ESI-TOF), $m / z[\mathrm{M}+\mathrm{H}]^{+}$ calcd for $\mathrm{C}_{10} \mathrm{H}_{12} \mathrm{~F}_{3} \mathrm{~N}_{4} \mathrm{O} 261.0958$, found 261.0965.

3-(Trifluoromethyl)-2H-benzo[e][1,2,4]thiadiazine 1,1-dioxide (10). A prepared solution of the trifluoroacetic acid $(0.44 \mathrm{~mL}, 5.69$ mmol, 1.0 equiv) in T3P $(50 \% \mathrm{w} / \mathrm{w}$ in toluene, $3.45 \mathrm{~mL}, 5.69 \mathrm{mmol}$, 1.0 equiv) was added dropwise to a suspension of 2 -aminobenzensulfonamide $9(1.0 \mathrm{~g}, 5.69 \mathrm{mmol}, 1.0$ equiv) in toluene (4 $\mathrm{mL})$ at rt. The reaction mixture was heated to $70{ }^{\circ} \mathrm{C}$ for $15 \mathrm{~h}$ and then additional T3P ( $50 \% \mathrm{w} / \mathrm{w}$ in toluene, $1.70 \mathrm{~mL}, 2.85 \mathrm{mmol}, 0.5$ equiv) added and further stirred at reflux for $24 \mathrm{~h}$. After completion of the reaction, the cooled reaction mixture was diluted with EtOAc $(20 \mathrm{~mL})$, quenched by dropwise addition of aq. sat. $\mathrm{NaHCO}_{3}(20 \mathrm{~mL}$, Caution! Gas evolution) followed by extraction with EtOAc $(20 \mathrm{~mL})$. Collected organic phases were washed with brine $(25 \mathrm{~mL})$, dried over anhydrous $\mathrm{MgSO}_{4}$, filtered and evaporated under reduced pressure to afford the crude compound as a light brown solid which was purified by trituration with TBME $(10 \mathrm{~mL})$ to yield 10 as an off white solid $(0.33$ g, 23\%). mp $154{ }^{\circ} \mathrm{C} .{ }^{1} \mathrm{H}$ NMR (500 MHz, DMSO) $\delta 7.78$ (d, $J=8.0$ $\mathrm{Hz}, 1 \mathrm{H}), 7.62(\mathrm{t}, J=7.0 \mathrm{~Hz}, 1 \mathrm{H}), 7.42(\mathrm{~d}, J=7.8 \mathrm{~Hz}, 2 \mathrm{H}), 3.37(\mathrm{br}$ $\mathrm{s}, 1 \mathrm{H}) .{ }^{13} \mathrm{C}$ NMR $(125 \mathrm{MHz}, \mathrm{DMSO}) \delta 147.8(\mathrm{~m}), 140.8,132.8$, $126.8,123.9,123.4,123.2,119(\mathrm{~m}) .{ }^{19} \mathrm{~F}$ NMR $(375 \mathrm{MHz}, \mathrm{DMSO}) \delta$ -70.70 . MS $[\mathrm{M}-\mathrm{H}]^{+} 249.04 \mathrm{~m} / z$. IR $1633(\mathrm{~m}) ; 1571(\mathrm{~s}), 1540(\mathrm{~s})$, 1471 (s), 1292 (s), 951 (s), 766 (s) $\mathrm{cm}^{-1}$. (ESI-TOF), $m / z[\mathrm{M}+\mathrm{H}]^{+}$ calcd for $\mathrm{C}_{8} \mathrm{H}_{6} \mathrm{~F}_{3} \mathrm{~N}_{2} \mathrm{O}_{2} \mathrm{~S}$ 251.0097, found 251.0000.

2-(Trifluoromethyl)quinazolin-4-(3H)-one (11). ${ }^{17}$ To a suspension of $\mathrm{AlCl}_{3}(0.35 \mathrm{~g}, 2.46 \mathrm{mmol}, 1.5$ equiv) in toluene $(4 \mathrm{~mL})$ was added at rt 3-benzyl-2-(trifluoromethyl)quinazolin-4-( $3 H)$-one (4a) (0.5 g, $1.64 \mathrm{mmol}, 1.0$ equiv) in one portion. After $0.5 \mathrm{~h}$ the reaction mixture was diluted with EtOAc $(15 \mathrm{~mL})$ and carefully quenched by dropwise addition of aq. sat. $\mathrm{NaHCO}_{3}(10 \mathrm{~mL})$. After extracting with EtOAc $(15 \mathrm{~mL})$, collected organic phase was washed with water and brine (10 $\mathrm{mL}$, respectively), dried over $\mathrm{MgSO}_{4}$, filtered and evaporated under reduced pressure to afford the crude compound as a white wet solid. Pure 11 was obtained after trituration with TBME $(4 \mathrm{~mL})$ as a white powder $(0.32 \mathrm{~g}, 91 \%) . \mathrm{mp} 111{ }^{\circ} \mathrm{C} .{ }^{1} \mathrm{H}$ NMR $\left(500 \mathrm{MHz}, \mathrm{CDCl}_{3}\right) \delta$ $8.38(\mathrm{~d}, J=8.9 \mathrm{~Hz}, 1 \mathrm{H}), 7.71-7.74(\mathrm{~m}, 2 \mathrm{H}), 7.39\left(\mathrm{td}, J_{1}=7.7 \mathrm{~Hz}, J_{2}\right.$ $=1.0 \mathrm{~Hz}, 1 \mathrm{H}) \cdot{ }^{13} \mathrm{C}$ NMR $\left(125 \mathrm{MHz}, \mathrm{CDCl}_{3}\right) \delta 155.1(\mathrm{q}, J=38.5$ $\mathrm{Hz}), 137.4,134.5,132.8,126.5,121.9,115.3,115.3(\mathrm{q}, J=288.7 \mathrm{~Hz})$, 103.8. ${ }^{19}$ F NMR (375 MHz, DMSO) $\delta-69.33$. MS $[\mathrm{M}+\mathrm{H}]^{+} 215.15$ $\mathrm{m} / z$. The analytical data are consistent with reported data.

4-Chloro-2-(trifluoromethyl)quinazoline (12). ${ }^{18} \mathrm{POCl}_{3}(1.02 \mathrm{~mL}$, $11.1 \mathrm{mmol}, 0.8$ equiv) was added at $95{ }^{\circ} \mathrm{C}$ to a white suspension of 11 $(2.96 \mathrm{~g}, 13.8 \mathrm{mmol}, 1.0$ equiv) in toluene $(18 \mathrm{~mL})$ and DMF $(1.6 \mathrm{~mL}$, $20.7 \mathrm{mmol}, 1.5$ equiv). After $30 \mathrm{~min}$, the mixture was cooled to $0{ }^{\circ} \mathrm{C}$ with an ice-water bath and quenched with $25 \mathrm{~mL}$ of water. The aqueous layer was extracted with EtOAc $(25 \mathrm{~mL})$ and collected organic phase was washed with water $(15 \mathrm{~mL})$, dried over $\mathrm{MgSO}_{4}$, filtered and evaporated under reduced pressure yielding chloroquinazoline 12 as a white solid $(2.83 \mathrm{~g}, 88 \%)$ of sufficient purity to be used as such in the next steps. mp $63{ }^{\circ} \mathrm{C}$ (DSC). ${ }^{1} \mathrm{H}$ NMR $(500 \mathrm{MHz}$, $\left.\mathrm{CDCl}_{3}\right) \delta 8.41\left(\mathrm{dd}, J_{1}=0.8 \mathrm{~Hz}, J_{2}=8.4 \mathrm{~Hz}, 1 \mathrm{H}\right), 8.26(\mathrm{~d}, J=8.5 \mathrm{~Hz}$, $1 \mathrm{H}), 8.14(\mathrm{~m}, 1 \mathrm{H}), 7.93(\mathrm{~m}, 1 \mathrm{H}) .{ }^{13} \mathrm{C} \mathrm{NMR}\left(125 \mathrm{MHz}, \mathrm{CDCl}_{3}\right) \delta$ $164.3,151.6(\mathrm{q}, J=37.6 \mathrm{~Hz}), 150.5,136.2,131.1,129.6,126.1,124.2$, $118.2(\mathrm{q}, J=275.7 \mathrm{~Hz}) \cdot{ }^{19} \mathrm{~F}$ NMR $\left(375 \mathrm{MHz}, \mathrm{CDCl}_{3}\right) \delta-69.98$. MS $[\mathrm{M}-\mathrm{H}]^{+}+\mathrm{H}_{2} \mathrm{O}$ 212.98. The analytical data are consistent with reported data.

4-Isopropyl-2-(trifluoromethyl)quinazoline (13). To a solution of $12\left(0.5 \mathrm{~g}, 2.15 \mathrm{mmol}, 1.0\right.$ equiv) in $\operatorname{dry} \mathrm{THF}(5 \mathrm{~mL})$ at $-20{ }^{\circ} \mathrm{C}$ was added dropwise a solution of $i$-propylmagnesium chloride- $\mathrm{LiCl}$ complex $14 \%$ in THF $(2.46 \mathrm{~mL}, 2.26 \mathrm{mmol}, 1.05$ equiv). The reaction mixture was stirred at this temperature for $15 \mathrm{~min}$, and at 0 ${ }^{\circ} \mathrm{C}$ iPrOAc $(10 \mathrm{~mL})$ added followed by aq. $20 \%$ citric acid $(5 \mathrm{~mL})$. Collected organic phases were washed with water $(2 \times 20 \mathrm{~mL})$, dried over $\mathrm{MgSO}_{4}$, filtered and evaporated under reduced pressure to afford compound 13 as a yellow liquid $(0.42 \mathrm{~g}, 81 \%) .{ }^{1} \mathrm{H}$ NMR $(500 \mathrm{MHz}$, DMSO) $\delta 8.57(\mathrm{~d}, J=8.4 \mathrm{~Hz}, 1 \mathrm{H}), 8.15-8.22(\mathrm{~m}, 2 \mathrm{H}), 7.96(\mathrm{~m}, 1$ $\mathrm{H}), 4.12-4.20(\mathrm{~m}, 1 \mathrm{H}), 1.39(\mathrm{~d}, J=6.7 \mathrm{~Hz}, 6 \mathrm{H}) .{ }^{13} \mathrm{C}$ NMR $(125$ MHz, DMSO) $\delta 179.1,151.3(\mathrm{q}, J=35.1 \mathrm{~Hz}), 149.3,136.0,130.7$, 129.6, 125.8, 123.3, $119.4(\mathrm{q}, \mathrm{J}=275.6 \mathrm{~Hz}), 31.1,22.1 .{ }^{19} \mathrm{~F}$ NMR $(375$ MHz, DMSO) $\delta-68.68$. MS $[\mathrm{M}+\mathrm{H}]^{+} 241.24$. IR $2975(\mathrm{~m}), 1617$ (m), 1399 (m), 1195 (s), 1169 (s), 1112 (s), 917 (s), 766 (s), 714 (m) $\mathrm{cm}^{-1}$. (ESI-TOF), $\mathrm{m} / z[\mathrm{M}+\mathrm{H}]^{+}$calcd for $\mathrm{C}_{12} \mathrm{H}_{12} \mathrm{~F}_{3} \mathrm{~N}_{2}$ 241.0947, found 241.0952 .

$\mathrm{N}$-(3-Chloro-4-fluorophenyl)-2-(trifluoromethyl)quinazolin-4amine (15). A mixture of 12 (1.0 g, $4.3 \mathrm{mmol}, 1.0$ equiv), 3-chloro-4fluoroaniline (14) $(0.64 \mathrm{~g}, 4.3 \mathrm{mmol}, 1.0$ equiv) and DIPEA $(1.12 \mathrm{~mL}$, $6.45 \mathrm{mmol}, 1.5$ equiv) in toluene $(10 \mathrm{~mL})$ was heated at reflux for 5.5 h. The reaction mixture was cooled to $\mathrm{rt}$, diluted with $10 \mathrm{~mL}$ of $i$ PrOAc and aq. citric acid $20 \%(5 \mathrm{~mL})$. After phase split, the aqueous phase was extracted with $i \operatorname{PrOAc}(10 \mathrm{~mL})$ and collected organic phases dried over $\mathrm{MgSO}_{4}$, filtered and evaporated under reduced pressure to afford crude compound as a brown tick oil which was purified by trituration with $\mathrm{MeCN}(4 \mathrm{~mL})$ yielding product 15 as a white powder $(1.04 \mathrm{~g}, 71 \%) . \mathrm{mp} 127{ }^{\circ} \mathrm{C} .{ }^{1} \mathrm{H}$ NMR $(500 \mathrm{MHz}$, DMSO) $\delta 10.38(\mathrm{~s}, 1 \mathrm{H}), 8.66(\mathrm{~d}, J=8.1 \mathrm{~Hz}, 1 \mathrm{H}), 8.25\left(\mathrm{dd}, J_{1}=2.6\right.$ $\left.\mathrm{Hz}, J_{2}=6.8 \mathrm{~Hz}, 1 \mathrm{H}\right), 7.97-8.04(\mathrm{~m}, 2 \mathrm{H}), 7.83-7.89(\mathrm{~m}, 2 \mathrm{H}), 7.53$ $(\mathrm{t}, \mathrm{J}=9.1 \mathrm{~Hz}, 1 \mathrm{H}) .{ }^{13} \mathrm{C}$ NMR $(125 \mathrm{MHz}, \mathrm{DMSO}) \delta 159.1,155.3$, $153.3,151.4(\mathrm{q}, J=34.8 \mathrm{~Hz}), 149.3,136.2,134.9,129.1(\mathrm{~d}, J=14.4$ $\mathrm{Hz}), 124.5,123.8,123.2(\mathrm{~d}, J=7.1 \mathrm{~Hz}), 119.5(\mathrm{~d}, J=18.4 \mathrm{~Hz}), 119.3$ $(\mathrm{m}), 117.3(\mathrm{~d}, J=21.9 \mathrm{~Hz}), 115.6 .{ }^{19} \mathrm{~F}$ NMR $(375 \mathrm{MHz}, \mathrm{DMSO}) \delta$ $-69.68,-121.51$. MS $[\mathrm{M}+\mathrm{H}]^{+}$342.07. IR $1920(\mathrm{w}), 1620(\mathrm{~m}), 1541$ (s), $1492(\mathrm{~s}), 1180(\mathrm{~s}), 1081(\mathrm{~m}), 960(\mathrm{~s}), 781(\mathrm{~s}), \mathrm{cm}^{-1}$. (ESI-TOF), $\mathrm{m} / z[\mathrm{M}+\mathrm{H}]^{+}$calcd for $\mathrm{C}_{15} \mathrm{H}_{9} \mathrm{ClF}_{4} \mathrm{~N}_{3} 342.0416$, found 342.0428 .

4-Methoxy-2-(trifluoromethyl)quinazoline (16). NaOMe $25 \% \mathrm{w} /$ $\mathrm{w}$ in $\mathrm{MeOH}(0.37 \mathrm{~mL}, 1.61 \mathrm{mmol}, 1.5$ equiv $)$ was added at $\mathrm{rt}$ to a solution of $12(0.25 \mathrm{~g}, 1.07 \mathrm{mmol}, 1.0$ equiv) in toluene $(2.5 \mathrm{~mL})$. After $1 \mathrm{~h}$ at $\mathrm{rt}$, the resulting suspension was diluted with $i \operatorname{PrOAc}(10$ $\mathrm{mL}$ ) and aq. $20 \%$ citric acid $(5 \mathrm{~mL})$. Aqueous phase was extracted once with $i \operatorname{PrOAc}(10 \mathrm{~mL})$ and collected organic phases dried over $\mathrm{MgSO}_{4}$, filtered and evaporated under reduced pressure to afford crude compound which was purified by trituration with $i \mathrm{PrOH}(2 \mathrm{~mL})$ where the product was recovered from the mother liquors by evaporation of the solvent under vacuum to yield product 16 as a white solid (0.24 g, 98\%). mp $98{ }^{\circ} \mathrm{C} .{ }^{1} \mathrm{H}$ NMR (500 MHz, DMSO) $\delta$ $8.29(\mathrm{~m}, 1 \mathrm{H}), 8.12(\mathrm{~m}, 2 \mathrm{H}), 7.88(\mathrm{~m}, 1 \mathrm{H}), 4.23(\mathrm{~s}, 3 \mathrm{H}) .{ }^{13} \mathrm{C}$ NMR 
(125 MHz, DMSO) $\delta 168.7,151.0(\mathrm{q}, J=36 \mathrm{~Hz}), 149.9,135.9,130.3$, $128.5,124.1,120.2(\mathrm{q}, J=276 \mathrm{~Hz}), 116.5,55.8 .{ }^{19} \mathrm{~F}$ NMR $(375 \mathrm{MHz}$, DMSO) $\delta$-69.23. MS $[\mathrm{M}+\mathrm{H}]^{+}$229.19. IR $3495(\mathrm{w}), 1698(\mathrm{~m})$, $1571(\mathrm{~m}), 1385$ (s), 1259 (m), 1159 (s), 1135 (s), 1101 (s), 727 (s); $683(\mathrm{~s}) \mathrm{cm}^{-1}$. (ESI-TOF), $\mathrm{m} / z[\mathrm{M}+\mathrm{H}]^{+}$calcd for $\mathrm{C}_{10} \mathrm{H}_{8} \mathrm{~F}_{3} \mathrm{~N}_{2} \mathrm{O}$ 229.0583 , found 229.0589 .

\section{ASSOCIATED CONTENT}

\section{S Supporting Information}

The Supporting Information is available free of charge on the ACS Publications website at DOI: 10.1021/acs.joc.8b00389.

NMR spectra, HPLC analysis, MS and UV spectra for compound 1 (PDF)

\section{AUTHOR INFORMATION}

\section{Corresponding Author}

*E-mail: simone.tortoioli@idorsia.com. Phone: +41 5884403 55.

\section{ORCID}

Simone Tortoioli: 0000-0002-5652-4124

\section{Present Address}

${ }^{\S}$ Firmenich SA, Route de La Plaine 125, 1283 La Plaine, Switzerland.

Notes

The authors declare no competing financial interest.

\section{ACKNOWLEDGMENTS}

We are grateful to Dr. Gabriel Schäfer for proofreading the manuscript and for valuable comments. The authors would like to thank Jürgen Seifert for IR measurements, François Le Goff and Marco Caldarone for HRMS measurements, Julien Grimont for NMR support, Markus von Raumer and his team for DSC measurements. Haute école d'ingénierie et d'architecture de Fribourg is also thanked for financial support. Finally, Dr. Martin Bolli is thanked for reviewing the manuscript.

\section{REFERENCES}

(1) (a) Filler, R.; Kobayashi, Y.; Yagupolskii, L. M. Organofluorine Compounds in Medicinal Chemistry and Biomedical Applications; Elsevier: Amsterdam, 1993. (b) Kirsch, P. Modern Fluoroorganic Chemistry: Synthesis, Reactivity, Applications; Wiley-VCH, 2004. (c) Welch, J. T. Tetrahedron 1987, 43, 3123-3197.

(2) (a) Ni, C.; Hu, J. Chem. Soc. Rev. 2016, 45, 5441-5454. (b) Organo-Fluorine Compounds. Methods of Organic Chemistry; Houben-Weyl, E10; Thieme Verlag: Stuttgart, 1999. (c) Groult, H.; Leroux, F.; Tressaud, A. Modern Synthetic Processes and Reactivity of Fluorinated Compounds; Elsevier Science: London, 2017. (d) Ma, J. A.; Cahard, D. J. Fluorine Chem. 2007, 128, 975-996. (trifluoromethylation). (e) Yang, X.; Wu, T.; Phipps, R. J.; Toste, F. D. Chem. Rev. 2015, 115, 826-870 (fluorinations and trifluoromethylation)..

(3) (a) Beatty, J. W.; Douglas, J. J.; Cole, K. P.; Stephenson, C. R. J. Nat. Commun. 2015, 6, 7919-7925. (b) Steiner, H. Chimica Oggi $\mathbf{2 0 1 5}, 33,26-37$. (c) Still, TFA remains a highly corrosive compound.

(4) (a) Chen, M.; Buchwald, S. L. Angew. Chem., Int. Ed. 2013, 52, 11628-11631. (potassium trifluoroacetate). (b) Carr, G. E.; Chambers, R. D.; Holmes, T. F.; Parker, D. G. J. Chem. Soc., Perkin Trans. 1 1988, 921-926. (sodium trifluoroacetate). (c) Li, Y.; Chen, T.; Wang, H.; Zhang, R.; Jin, K.; Wang, X.; Duan, C. Synlett 2011, 12, 1713-1716. (sodium trifluoroacetate). (d) Langlois, B. R.; Roques, N. J. Fluorine Chem. 2007, 128, 1318-1325. (methyl trifluoroacetate). (e) Schareina, T.; Wu, X.-F.; Zapf, A.; Cotté, A.; Gotta, M.; Beller, M. Top. Catal. 2012, 55, 426-431 (methyl trifluoroacetate)..
(5) Representative examples: (a) Alberti, M. N.; Polyhach, Y. T.; Manolis, D.; Tödtli, L.; Jeschke, G.; Diederich, F. Chem. - Eur. J. 2016, 22, 10194-10202. (b) Sinn, S.; Biedermann, F.; De Cola, L. Chem. Eur. J. 2017, 23, 1965-1971. (c) Schäfer, G.; Ahmetovic, M.; Abele, S. Org. Lett. 2017, 19, 6578-6581. (d) Fan, K. W.; Peterson, M. B.; Ellersdorfera, P.; Granville, A. M. RSC Adv. 2016, 6, 25203-25214. (e) Wang, Z.-X.; Ma, Q.-W. J. Heterocyclic Chem. 2015, 52, 18931896. (f) Hoover, D. J.; Witter, K. G. Pfizer Products Inc. WO2008/ 004117, 2008. (g) Culshaw, A. J.; Brain, C. T.; Dziadulewicz, E. C.; Edwards, L.; Hart, T. W.; Ritchie, T. J. Novartis Pharma WO 2007/ 065888, 2007. (h) Lechel, T.; Gerhard, M.; Trawny, D.; Brusilowskij, B.; Schefzig, L.; Zimmer, R.; Rabe, J. P.; Lentz, D.; Schalley, C. A.; Reissig, H.-U. Chem. - Eur. J. 2011, 17, 7480-7491. (TFA). (i) René, O.; Souverneva, A.; Magnuson, S. R.; Fauber, B. P. Tetrahedron Lett. 2013, 54, 201-204. (j) Wang, Z.-X.; Zhang, T.-F.; Ma, Q.-W.; Ni, W.G. Synthesis 2014, 46, 3309-3314. (k) Hazelton, J. C.; Iddon, B.; Suschitzky, H.; Woolley, L. H. Tetrahedron 1995, 51, 10771-10794. (l) Roberts, L. R.; Bradley, P. A.; Bunnage, M. E.; England, K. S.; Fairman, D.; Fobian, Y. M.; Fox, D. N. A.; Gymer, G. E.; Heasley, S. E.; Molette, J.; Smith, G. L.; Schmidt, M. A.; Tones, M. A.; Dack, K. N. Bioorg. Med. Chem. Lett. 2011, 21, 6515-6518.

(6) Representative examples: (a) Bisson, J.; Dehaudt, J.; Charbonnel, M.-C.; Guillaneux, D.; Miguirditchian, M.; Marie, C.; Boubals, N.; Guy, D.; Blot, V.; Dubreuil, D. Chem. - Eur. J. 2014, 20, 7819-7829. (trifluoroacetic anhydride). (b) Martens, S. C.; Zschieschang, U.; Wadepohl, H.; Klauk, H.; Gade, L. H. Chem. - Eur. J. 2012, 18, 34983509. (trifluoroacetic anhydride). (c) Masahiro, O.; Kenji, F.; Kenji, U.; Norimitsu, H.; Daisuke, I.; Hideki, H.; Noriaki, H. Mitsubishi Tanabe Pharma Corporation TW2016/2115 A, 2016 (trifluoroacetic anhydride). (d) Overberger, C. G.; Michelotti, F. W.; Carabateas, P. M. J. Am. Chem. Soc. 1957, 79, 941-944. (ethyl trifluoroacetate). (e) Sielecki, T. M.; Johnson, T. L.; Liu, J.; Muckelbauer, J. K.; Grafstrom, R. H.; Cox, S.; Boylan, J.; Burton, C. R.; Chen, H.; Smallwood, A.; Chang, C.-H.; Boisclair, M.; Benfield, P. A.; Trainora, G. L.; Seitza, S. P. Bioorg. Med. Chem. Lett. 2001, 11, 1157-1160. (ethyl trifluoroacetate). (f) Grewal, G.; Hennessy, E.; Kamhi, V.; Li, D.; Lyne, P.; Oza, V.; Saleh, J. C.; Su, Q.; Yang, B. Astrazeneca UK Limited WO 2008/056150 A1, 2008 (ethyl trifluoroacetate).

(7) (a) Lechel, T.; Dash, J.; Hommes, P.; Lentz, D.; Reissig, H.-U. J. Org. Chem. 2010, 75, 726-732. (b) Dash, J.; Lechel, T.; Reissig, H.-U. Org. Lett. 2007, 9, 5541-5544. (c) Song, X.-J.; Yang, P.; Gao, H.; Wang, Y.; Dong, X.-G.; Tan, X.-H. Chin. Chem. Lett. 2014, 25, 10061010. (d) Ding, J.; Gao, J.; Wu, H.; Huang, X.; Yuan, C. Synth. Commun. 2005, 35, 511-519. (e) Rahirnizadeh, M.; Tavallai, Z.; Bakavoli, M. Indian J. Chem. 2004, 43, 679-681.

(8) (a) Kumar, D.; Jadhavar, P. S.; Nautiyal, M.; Sharma, H.; Meena, P. K.; Adane, L.; Pancholia, S.; Chakraborti, A. K. RSC Adv. 2015, 39, 30819-30825. (b) Bipransh Kumar Tiwary, B. K.; Pradhan, K.; Nand, A. K.; Chakraborty, R. J. Chem. Biol. Ther. 2015, 104, 1. (c) Bouley, R.; Ding, D.; Peng, Z.; Bastian, M.; Lastochkin, E.; Song, W.; Suckow, M. A.; Schroeder, V. A.; Wolter, W. R.; Mobashery, S.; Chang, M. J. Med. Chem. 2016, 59, 5011-5021. (d) Alam, M. J.; Alam, O.; Naim, M. J.; Alam, P. Int. J. Ad. Res. 2015, 3, 1656-1664. (e) For a recent review on synthetic strategies: Rohokale, R. S.; Kshirsagar, U. A. Synthesis 2016, 48, 1253-1268.

(9) (a) Gigante, A.; Canela, M.-D.; Delang, L.; Priego, E.-M.; Camarasa, M.-J.; Querat, G.; Neyts, J.; Leyssen, P.; Pérez-Pérez, M.-J. J. Med. Chem. 2014, 57, 4000-4008. (b) Bhonoah, Y.; Elliott, A. C.; Gaulier, S.; Ling, K.; Mitchell, G.; Morris, J. A.; Rzepa, P. R.; Viner, R. C. Syngenta Limited WO2013/50421 A1, 2013. (c) Madge, D.; Chan, F.; John, D. E.; Edwards, S. D.; Blunt, R.; Hartzoulakis, B.; Brown, L. Xention Limited WO2013/72694 A1, 2013. (d) Okuda, K.; Takarada, S.; Hirota, T.; Sasaki, K. J. Heterocyclic Chem. 2015, 52, 780-792. (e) Zhu, J.; Xie, H.; Chen, Z.; Li, S.; Wu, Y. Org. Biomol. Chem. 2012, 10, 516-523. (f) Bergman, J.; Bergman, S. J. Org. Chem. 1985, 50, $1246-1255$.

(10) For a recent application of T3P: Bannwart, L.; Abele, S.; Tortoioli, S. Synthesis 2016, 48, 2069-2078. 
(11) (a) Desroses, M.; Scobie, M.; Helleday, T. New J. Chem. 2013, 37, 3595-3597. (b) Raghavendra, G. M.; Kumar, C. S. P.; Suresha, G. P.; Rangappa, K. S.; Mantelingu, K. Chin. Chem. Lett. 2015, 26, $963-$ 968.

(12) Representative examples: (a) Diener, M. E.; Metrano, A. J.; Kusano, S.; Miller, S. J. J. Am. Chem. Soc. 2015, 137, 12369-12377. (b) Wu, X. F.; Neumann, H.; Beller, M. Chem. - Eur. J. 2012, 18, 12599-12602. (c) Borisek, J.; Vizovisek, M.; Sosnowski, P.; Turk, B.; Turk, D.; Mohar, B.; Novic, M. J. Med. Chem. 2015, 58, 6928-6937. (d) Kanth, S. R.; Maitraie, D.; Reddy, G. V.; Narsaiah, B.; Rao, P. S. Heterocycles 2005, 65, 1415-1423.

(13) Only T3P derived adducts were detected. Alternatively, primary amide 3a was obtained in $41 \%$ yield with T3P from a solution of $\mathrm{NH}_{3}$ in dioxane after $24 \mathrm{~h}$ at reflux in an autoclave reactor. We speculated that ammonia can competitively react with T3P becoming therefore unavailable for amidation.

(14) Dimers were not isolated and their structure determined on the basis of LC-MS analyses. Although increased acidity of the amino group on substrates $\mathbf{1 j}-\mathbf{l}, \mathbf{o}$ is foreseen $\left(\mathrm{p} K_{\mathrm{a}}\right.$ : 4.64 for aniline, 4.11 for naphthalen-2-amine, 3.14 for 2-aminopyrazine and 2.45 for 3 nitroaniline, source: http://www.chem.wisc.edu/areas/reich/ pkatable/pKa_compilation-1-Williams.pdf), there is no clear explanation for the occurrence of this side-reaction only with substrates $\mathbf{1 j}-$ 1,o.

(15) The yield refers to a nonoptimized strategy I protocol where incomplete conversion was observed. It is well probable that to have a more productive process, optimization (including reverting the order of addition as for strategy II) could be performed. For representative examples see: (a) Antolak, S. A.; Yao, Z.-K.; Richoux, G. M.; Slebodnick, C.; Carlier, P. R. Org. Lett. 2014, 16, 5204-5207. (b) Witt, A.; Gustavsson, A.; Bergman, J. J. Heterocyclic Chem. 2003, 40, 29-35. (c) Luo, S.-P.; Geng, H.; Wang, Y.; Huang, P.-Q. Chin. J. Chem. 2015, 33, 646-654. (d) Al-Said, N. H.; Shawakfeh, K. Q.; Ibrahim, M. I.; Tayyem, S. H. Arkivoc 2010, ix, 282-292. (e) Leit, S.; Wahhb, A.; Allan, M.; Smil, D.; Tessier, P.; Deziel, R.; Chantigny, Y. A. Methylgene Inc. WO2007/22638, 2007. (f) Roos, G. H. P.; Dastlik, K. A. Heterocycles 2003, 60, 2023-2044.

(16) (a) Basavaprabhu; Vishwanatha, T. M.; Panguluri, N. R.; Sureshbabu, V. V. Synthesis 2013, 45, 1569-1601. (b) Valeur, E.; Bradley, M. Chem. Soc. Rev. 2009, 38, 606-631. (c) Waghmare, A. A.; Hindupur, R. M.; Pati, H. N. Ref. J. Chem. 2014, 4, 53-131.

(17) Venkatesh, R.; Kasaboina, S.; Gaikwad, H. K.; Janardhan, S.; Bantu, R.; Nagarapu, L.; Sastry, G. N.; Banerjee, S. K. Eur. J. Med. Chem. 2015, 96, 22-29.

(18) Storz, T.; Heid, R.; Zeldis, J.; Hoagland, S. M.; Rapisardi, V.; Hollywood, S.; Morton, G. Org. Process Res. Dev. 2011, 15, 918-924. 